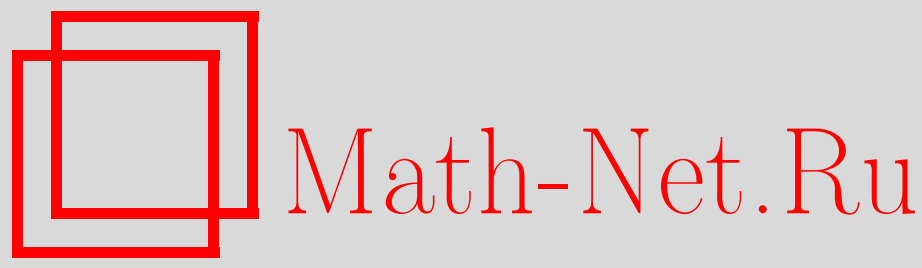

М. Н. Дубинин, Е. Ю. Петрова, Высокотемпературный потенциал Хиггса двухдублетной модели в рамках теории катастроф, ТМФ, 2015, том 184, номер 2, 315-337

DOI: https://doi.org/10.4213/tmf8814

Использование Общероссийского математического портала Math-Net.Ru подразумевает, что вы прочитали и согласны с пользовательским соглашением http://www . mathnet.ru/rus/agreement

Параметры загрузки:

IP: 3.85 .183 .62

26 апреля 2023 г., 13:08:55

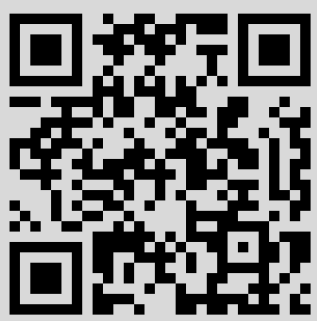




\title{
ФИЗИКА
}

Том 184, № 2

август, 2015

\section{ВЫСОКОТЕМПЕРАТУРНЫЙ ПОТЕНЦИАЛ ХИГГСА ДВУХДУБЛЕТНОЙ МОДЕЛИ В РАМКАХ ТЕОРИИ КАТАСТРОФ}

\begin{abstract}
Рассматривается общий случай температурной эволюции двухдублетного потенциала Хиггса минимальной суперсимметричной стандартной модели, когда вакуумные конденсаты хиггсовских дублетов произвольно перемещаются по поверхности равновесия (или поверхности экстремумов), проходя через области бифуркаций. В рамках теории катастроф двухдублетный потенциал Хиггса минимальной суперсимметричной стандартной модели представляется специальным случаем потенциала градиентной системы. Получены нелинейные преобразования вакуумных конденсатов указанной модели, приводящие двухдублетный потенциал к канонической форме, а также функции-катастрофы типа $A_{3}$ и $A_{5}$, соответствующие электрослабому фазовому переходу первого рода в данной модели.
\end{abstract}

Ключевые слова: двухдублетная модель, минимальная суперсимметричная стандартная модель, потенциал Хиггса при конечных температурах, электрослабый фазовый переход, канонические формы теории катастроф.

DOI: $10.4213 / \operatorname{tmf} 8814$

\section{1. ВВЕДЕНИЕ}

Согласно имеющимся представлениям об эволюции ранней Вселенной в рамках концепции Большого взрыва в первичной космической плазме, образовавшейся во время "горячей стадии", могли происходить фазовые переходы, связанные с эволюцией основного состояния Вселенной при уменьшении температуры от нескольких сотен ГэВ до нескольких ГэВ. При температуре порядка нескольких сотен МэВ мог происходить фазовый переход от кварк-глюонной плазмы к адронной материи, сопровождаемый нарушением киральной симметрии. При температурах порядка $10^{2}$ ГэВ (порядка $10^{15} \mathrm{~K}$ ) или существенно больших мог происходить электрослабый фазовый переход [1] из состояния с нулевым вакуумным средним поля

${ }^{*}$ Научно-исследовательский институт ядерной физики им. Д. В. Скобельцына, Московский государственный университет им. М. В. Ломоносова, Москва, Россия.

E-mail: dubinin@theory.sinp.msu.ru

${ }^{\dagger}$ Физический факультет, Московский государственный университет им. М. В. Ломоносова, Москва, Россия. E-mail: petrova@theory.sinp.msu.ru 
(полей) Хиггса и безмассовыми калибровочными полями к состоянию с ненулевыми вакуумными конденсатами, сопровождаемый нарушением калибровочной симметрии $S U(2)_{L} \times U(1)_{Y}$ до электромагнитной симметрии $U(1)_{\mathrm{em}}$ и генерацией барионной асимметрии Вселенной [2]. В рамках термодинамического описания эффективный потенциал Хиггса стандартной модели (CM) представляет собой плотность свободной энергии плазмы фундаментальных фермионов и бозонов, а также скалярных частиц с достаточно малым самодействием ${ }^{1)}$. В СМ удается обеспечить фазовый переход первого рода лишь в области значений масс бозона Хиггса менее 50 ГэВ, что не отвечает наблюдаемому на LHC (Большом адронном коллайдере) скалярному резонансу с массой $m_{H}=125$ ГэВ [3], свойства которого хорошо соответствуют CM [4]. Отсутствие возможностей для электрослабого бариогенезиса в СМ можно обойти в ее расширениях, наиболее популярным из которых является минимальная суперсимметричная стандартная модель $(\mathrm{MCCM})^{2)}$.

В рамках рассматривающихся моделей эволюции Вселенной [5] "горячей стадии" предшествует инфляционная стадия, в ходе которой Вселенная становится однородной на космологических масштабах и с высокой точностью пространственно-плоской. Нетепловая инфляционная стадия завершается неравновесным разогревом, приводящим к первичной плазме с температурой, грубая оценка которой в обширном интервале $M_{\text {Planck }}-1$ ТэВ (масса Планка $M_{\text {Planck }}=1.2 \times 10^{19}$ ГэВ) соответствует длительности инфляции $10^{-43}-10^{-11}$ с, достаточной для расширения первичной области с размером порядка $1 / M_{\text {Planck }}$ до космологических масштабов, что обеспечивает решение проблемы космологического горизонта. Ненаблюдаемость магнитных монополей указывает на малую вероятность очень высоких температур, бо́льших или порядка масштаба Большого объединения $M_{\mathrm{GUT}} \sim 10^{16}$ ГэВ, это смещает оценки температуры плазмы ближе к нижней границе, но сохраняет очень большую степень неопределенности этой величины. Для более определенных заключений требуется теоретико-полевая модель ранней Вселенной, которая не определена.

Напомним, что в рамках имеющихся реализаций инфляционной космологической модели (наиболее известными примерами которой являются модели новой инфляици [6], хаотической инфлячии [7] и гибридной инфляиии [8]) вводится скалярное поле - инфлатон, потенциал которого дает основной вклад в плотность энергии ранней Вселенной. Инфляционные сценарии пространственно-плоской Вселенной реализуются для режима медленного скатывания, когда расширение обусловлено потенциальной энергией медленно меняющегося поля инфлатона. Этот процесс завершается высокочастотными осцилляциями инфлатона вблизи минимума потенциала, приводящими к интенсивному рождению взаимодействующих с ним частиц, и переходом в наиболее интересующую нас "горячую стадию". Весьма нетривиальной проблемой представляется идентификация слабовзаимодействующего инфлатона в рамках хорошо изученных в настоящее время калибровочных моделей теории поля, которая позволила бы обнаружить его в экпериментах на LHC и планируемых экспериментах на ILC (Международном линейном коллайдере), надежно вычислить температуру первичной плазмы и другие космологические наблюдаемые. Единственным кандидатом на роль инфлатона в рамках СМ является бозон Хиггса.

1) При нулевой температуре константа самодействия полей Хиггса в СМ $\lambda / 4=0.53 / 4=0.13$.

${ }^{2)}$ Возможно также, что барионная асимметрия генерируется не на "горячей стадии", а на термодинамически неравновесной постинфляционной стадии за счет других механизмов. 
Длительное время считалось, что бозон Хиггса не подходит на роль инфлатона, поскольку его константа самодействия слишком велика для генерации космологических возмущений плотности, согласующихся с наблюдениями, а также большие радиационные поправки к потенциалу Хиггса в СМ вызывали сомнения в возможности реализации достаточно длительного режима медленного скатывания. Однако эти сомнения были пересмотрены в последних работах [9], где хиггсовский сектор $\mathrm{CM}$ имеет неминимальное взаимодействие с гравитацией. Расширения СМ для объяснений осцилляций нейтрино и включения темной материи возможны за счет правого стерильного нейтрино. Интенсивные осцилляции инфлатона Хиггса вблизи минимума приводят к рождению калибровочных бозонов СМ, которые впоследствии аннигилируют в кварки и лептоны. Генерация барионной асимметрии происходит на стадии постинфляционного разогрева ${ }^{3)}$. Модель стандартного инфлатона Хиггса хорошо соответствует экспериментальным измерениям микроволнового космологического фона. Существенно, что в модели отсутствуют какие-либо масштабы "новой физики" (суперпартнеры, составленность) вплоть до масштаба $M_{\text {Planck }}$ вследствие чего необходим механизм контроля радиационных поправок.

Контроль радиационных поправок, как известно, надежно присутствует в МССМ и ее расширениях ${ }^{4}$. В МССМ квадратичные расходимости фермионных и бозонных петель сокращаются, сохраняя режим медленного скатывания для эффективного потенциала Хиггса. Появление многочисленных скалярных суперпартнеров кварков и лептонов и дополнительных (как правило, тяжелых) бозонов Хиггса создает основу идентификации одного из суперпартнеров или бозонов как инфлатона [10]. Хорошо определенная форма лагранжевых членов МССМ могла бы позволить явно рассчитать процессы разогрева и образования первичной плазмы МССМ. Эта возможность представляется ценной, она позволит в будущем исследовать как инфляционную, так и термодинамическую эволюцию ранней Вселенной на основе экстраполяций низкоэнергетической феноменологии частиц, изученной экспериментально на коллайдерах LHC и ILC.

В дальнейшем предполагается, что барионная асимметрия образуется на "горячей стадии". Обоснование очень малого значения наблюдаемого отношения плотности барионного числа к плотности энтропии $n_{\mathrm{B}} / n_{s} \sim 10^{-10}$ является одной из основных проблем космологии. Если первое из трех необходимых условий генерации барионной асимметрии (нарушение барионного числа) может выполняться в СМ за счет сфалерона, то два других условия (нарушение $C$ - и $C P$-инвариантности, а также отклонение от термодинамического равновесия) в рамках CM, по-видимому, не выполняются. Матрица Кабиббо-Кобаяши-Маскава дает неприемлемо малое для генерации асимметрии нарушение $C P$-инвариантности, а электрослабый фазовый переход первого рода, как уже упоминалось, имеет место в СМ при $m_{H} \leqslant 50$ ГэВ [2]. МССМ предоставляет возможности реализации электрослабого фазового перехода первого рода (рассматривавшегося многими авторами [11]), а также включает многочисленные новые источники нарушения $C P$-инвариантности. Вместе с тем исследование

\footnotetext{
3) Возможно, генерацию барионной асимметрии можно обеспечить и на "горячей стадии", например, за счет расширения левыми майорановскими изодублетами лептонов (лептогенезис).

4) K расширениям относится не только неминимальная МССМ (НМССМ), поскольку квантовые поправки к параметрам потенциала МССМ нарушают специфические соотношения между константами взаимодействия четырех скалярных полей $\lambda_{i}, i=1, \ldots, 7$, приводя к эффективному потенциалу общей двухдублетной модели хиггсовского сектора.
} 
фазового перехода в рамках МССМ существенно усложняется по сравнению со СМ вследствие того, что для эффективного $C P$-инвариантного потенциала (здесь параметры $\lambda_{i}, i=5,6,7$, при членах потенциала размерности 4 - действительные числа) нарушение симметрии в общем базисе скалярных полей описывается двумя вакуумными средними, а система бозонов Хиггса состоит из пяти скалярных частиц: $h, H$ ( $C P$-четные состояния), $A$ ( $C P$-нечетное состояние) и $H^{ \pm}$(заряженные состояния). Независимо от того, зависит двухдублетный потенциал МССМ от температуры или не зависит, его нужно правильно интерпретировать в терминах частиц (другими словами, согласованно преобразовать $S U(2)$-состояния скалярных полей двух изодублетов в массовый базис полей Хиггса) и согласованным образом ввести радиационные и/или температурные поправки.

Настоящая работа использует результаты работ [12], [13], где получено общее преобразование двухдублетного потенциала в массовый базис полей Хиггса и вычислен ресуммированный (типа Коулмена-Вайнберга) эффективный потенциал МССМ при нулевой температуре. Нетривиальное нелинейное преобразование калибровочного базиса эффективного потенциала Хиггса MCCM в массовый базис для скаляров включает два угла смешивания (обычно обозначаемых $\alpha$ и $\beta$ ) компонентных полей хиггсовских изодублетов. В работах [12] можно найти численное исследование однои двухпетлевых радиационных поправок в нескольких характерных областях пространства параметров МССМ для общего случая явно нарушающего $C P$-симметрию эффективного потенциала (параметры $\lambda_{i}, i=5,6,7$, при членах потенциала размерности 4 - комплексные числа), который учитывает нетривиальные смешивания в $F$ и $D$-членах нарушения суперсимметрии, анализ точности приближений, например только главных $D$-членов, и т.п. Самодействия трех и четырех бозонов Хиггса детально исследовались в [14]. В работах [15] описанный выше эффективный потенциал при нулевой температуре $T=0$ рассматривался как граничное условие для высокотемпературного эффективного двухдублетного потенциала МССМ, "погруженного" в первичную космологическую плазму. В симметричной космологической фазе после разогрева вакуумные средние $v_{1}(T)$ и $v_{2}(T)$ равны нулю. Вышеупомянутые преобразования позволяют явно определить температурную эволюцию наблюдаемых исходя из температурной эволюции эффективных параметров $\lambda_{i}(T)$, $i=1, \ldots, 7,-$ факторов при членах потенциала размерности 4 . Однопетлевые температурные поправки к параметрам $\lambda_{i}(T)$ вычислены для разных типов диаграмм в формализме мнимого времени, что позволяет согласованно определить углы смешивания, зависящие от температуры массы бозонов Хиггса и другие наблюдаемые.

Поскольку, в отличие от СM, в рамках двухдублетной модели нет наглядной трехмерной картины для потенциала Хиггса, для изучения термодинамической эволюции интересно применить имеющийся формализм теории катастроф, в рамках которого получена классификация форм функций-катастроф с известными свойствами в окрестностях неизолированных критических точек. Теорией катастроф (для наших целей достаточно элементарного изложения, см., например, [16]) называется раздел математики, изучающий общие виды зависимостей решений уравнений движения от параметров, которые содержатся в уравнениях движения. В рамках теории катастроф двухдублетный эффективный потенциал может описывать "градиентную систему" (где "силы" могут быть получены вычислением градиента потенциала), зависящую от двух переменных состояния (вакуумные средние хиггсовских 
изодублетов $v_{1}$ и $\left.v_{2}\right)$ и некоторого числа управляющих параметров $\lambda_{i}$, количество которых определяется спецификой модели (общая двухдублетная модель, двухдублетная модель с дискретной симметрией, модель хиггсовского сектора МССМ и т.д.). В рамках квантовой теории поля при конечных температурах эти параметры зависят от полей других частиц и температуры. Например, экстремальная поверхность (или поверхность равновесия) двухдублетного потенциала Хиггса ранней Вселенной определяется уравнениями

$$
\frac{\partial U\left(v_{i} ; T\right)}{\partial v_{j}}=0, \quad i, j=1,2,
$$

решения которых зависят от температуры и управляющих параметров, явно определяя тем самым превращение симметричной фазы в фазу с нарушенной симметрией (“спонтанное" нарушение симметрии).

\section{2. ПРЕДСТАВЛЕНИЯ ДЛЯ ДВУХДУБЛЕТНОГО ПОТЕНЦИАЛА}

Двухдублетный эффективный потенциал Хиггса в общем базисе имеет вид

$$
\begin{aligned}
U\left(\Phi_{1}, \Phi_{2}\right)=- & \mu_{1}^{2}\left(\Phi_{1}^{\dagger} \Phi_{1}\right)-\mu_{2}^{2}\left(\Phi_{2}^{\dagger} \Phi_{2}\right)-\mu_{12}^{2}\left(\Phi_{1}^{\dagger} \Phi_{2}\right)-\mu_{12}^{2}\left(\Phi_{2}^{\dagger} \Phi_{1}\right)+ \\
& +\lambda_{1}\left(\Phi_{1}^{\dagger} \Phi_{1}\right)^{2}+\lambda_{2}\left(\Phi_{2}^{\dagger} \Phi_{2}\right)^{2}+\lambda_{3}\left(\Phi_{1}^{\dagger} \Phi_{1}\right)\left(\Phi_{2}^{\dagger} \Phi_{2}\right)+\lambda_{4}\left(\Phi_{1}^{\dagger} \Phi_{2}\right)\left(\Phi_{2}^{\dagger} \Phi_{1}\right)+ \\
& +\frac{\lambda_{5}}{2}\left(\Phi_{1}^{\dagger} \Phi_{2}\right)\left(\Phi_{1}^{\dagger} \Phi_{2}\right)+\frac{\lambda_{5}}{2}\left(\Phi_{2}^{\dagger} \Phi_{1}\right)\left(\Phi_{2}^{\dagger} \Phi_{1}\right)+ \\
& +\lambda_{6}\left(\Phi_{1}^{\dagger} \Phi_{1}\right)\left(\Phi_{1}^{\dagger} \Phi_{2}\right)+{ }^{*}{ }_{6}\left(\Phi_{1}^{\dagger} \Phi_{1}\right)\left(\Phi_{2}^{\dagger} \Phi_{1}\right)+ \\
& +\lambda_{7}\left(\Phi_{2}^{\dagger} \Phi_{2}\right)\left(\Phi_{1}^{\dagger} \Phi_{2}\right)+{ }^{*}{ }_{7}\left(\Phi_{2}^{\dagger} \Phi_{2}\right)\left(\Phi_{2}^{\dagger} \Phi_{1}\right),
\end{aligned}
$$

где $\mu_{1}^{2}, \mu_{12}^{2}, \mu_{2}^{2}$ - параметры размерности $2\left(\Gamma \ni \mathrm{B}^{2}\right)$, а $\lambda_{1}, \ldots, \lambda_{7}$ - безразмерные управляющие параметры, зависящие от температуры. Параметры $\mu_{12}^{2}$ и $\lambda_{5}, \lambda_{6}, \lambda_{7}$ могут быть комплексными [12], [17], тогда эрмитов эффективный потенциал, включающий мнимые части, явно нарушает $C P$-инвариантность. В дальнейшем считаем $\mu_{12}^{2}$ и $\lambda_{5}$, $\lambda_{6}, \lambda_{7}$ действительными. Изодублеты комплексных скалярных полей

$$
\begin{gathered}
\Phi_{1}=\left(\begin{array}{c}
\phi_{1}^{+}(x) \\
\phi_{1}^{0}(x)
\end{array}\right)=\left(\begin{array}{c}
-i \omega_{1}^{+} \\
\frac{1}{\sqrt{2}}\left(v_{1}+\eta_{1}+i \chi_{1}\right)
\end{array}\right), \\
\Phi_{2}=\left(\begin{array}{c}
\phi_{2}^{+}(x) \\
\phi_{2}^{0}(x)
\end{array}\right)=\left(\begin{array}{c}
-i \omega_{2}^{+} \\
\frac{1}{\sqrt{2}}\left(v_{2}+\eta_{2}+i \chi_{2}\right)
\end{array}\right)
\end{gathered}
$$

имеют зависящие от температуры вакуумные средние

$$
\left\langle\Phi_{1}\right\rangle=\frac{1}{\sqrt{2}}\left(\begin{array}{c}
0 \\
v_{1}(T)
\end{array}\right), \quad\left\langle\Phi_{2}\right\rangle=\frac{1}{\sqrt{2}}\left(\begin{array}{c}
0 \\
v_{2}(T)
\end{array}\right),
$$

$v^{2}=v_{1}^{2}+v_{2}^{2}, v_{2} / v_{1}=\operatorname{tg} \beta$. Поля $\eta_{i}, \chi_{i}, i=1,2$, не являющиеся массовыми состояниями, осциллируют вблизи зависящего от температуры положения равновесия $v_{i}(T)$, $i=1,2$. Чтобы интерпретировать потенциал (1) в терминах частиц и получить 
массовые состояния пяти бозонов Хиггса $h, H, A, H^{ \pm}$и голдстоуновского бозона $G^{0}$, необходимо диагонализовать матрицу, составленную из вторых производных потенциала Хиггса по полям, т. е. осуществить два ортогональных преобразования, определяемых углами $\alpha, \beta$ (здесь и далее используются обозначения $\sin \alpha=s_{\alpha}$ и т. п.),

$$
\begin{array}{ll}
\eta_{1}=-s_{\alpha} h+c_{\alpha} H, & \chi_{1}=-s_{\beta} A+c_{\beta} G^{0}, \\
\eta_{2}=c_{\alpha} h+s_{\alpha} H, & \chi_{2}=c_{\beta} A+s_{\beta} G^{0}
\end{array}
$$

и минимизировать потенциал (1). Потенциал Хиггса (1) в пространстве вакуумных ожиданий, когда осцилляции полей "выключены", имеет вид

$$
\begin{aligned}
U_{\text {eff }}\left(v_{1}, v_{2}\right)=- & \frac{1}{2} \mu_{1}^{2} v_{1}^{2}-\frac{1}{2} \mu_{2}^{2} v_{2}^{2}-\mu_{12}^{2} v_{1} v_{2}+\frac{1}{4} \lambda_{1} v_{1}^{4}+ \\
& +\frac{1}{4} \lambda_{2} v_{2}^{4}+\frac{1}{4} \lambda_{345} v_{1}^{2} v_{2}^{2}+\frac{1}{2} \lambda_{6} v_{1}^{3} v_{2}+\frac{1}{2} \lambda_{7} v_{1} v_{2}^{3},
\end{aligned}
$$

где $\lambda_{345}=\lambda_{3}+\lambda_{4}+\lambda_{5}$. Условие $\nabla U=0$ определяет существование экстремума

$$
\begin{aligned}
& \mu_{1}^{2}=\lambda_{1} v_{1}^{2}+\lambda_{345} \frac{v_{2}^{2}}{2}-\mu_{12}^{2} \frac{v_{2}}{v_{1}}+\frac{1}{2}\left(3 \lambda_{6} v_{1} v_{2}+\lambda_{7} \frac{v_{2}^{3}}{v_{1}}\right) \\
& \mu_{2}^{2}=\lambda_{2} v_{2}^{2}+\lambda_{345} \frac{v_{1}^{2}}{2}-\mu_{12}^{2} \frac{v_{1}}{v_{2}}+\frac{1}{2}\left(\lambda_{6} \frac{v_{1}^{3}}{v_{2}}+3 \lambda_{7} v_{1} v_{2}\right) .
\end{aligned}
$$

Связи (5) определяют поверхность экстремумов (или поверхность равновесия), которую можно получить стандартным методом неопределенных множителей Лагранжа в форме

$$
U^{\lambda}\left(v_{1}, v_{2}\right)=-\frac{1}{4} \lambda_{1} v_{1}^{4}-\frac{1}{4} \lambda_{2} v_{2}^{4}-\frac{1}{4} \lambda_{345} v_{1}^{2} v_{2}^{2}-\frac{1}{2} \lambda_{6} v_{1}^{3} v_{2}-\frac{1}{2} \lambda_{7} v_{1} v_{2}^{3}
$$

или в эквивалентной форме, использующей $\mu_{1}^{2}, \mu_{2}^{2}, \mu_{12}^{2}$,

$$
U_{\mathrm{ex}}^{\mu}\left(v_{1}, v_{2}\right)=-\frac{1}{4} \mu_{1}^{2} v_{1}^{2}-\frac{1}{4} \mu_{2}^{2} v_{2}^{2}-\frac{1}{2} \mu_{12}^{2} v_{1} v_{2} .
$$

Наличие минимумов потенциала обеспечивается условием $\operatorname{det} U \geqslant 0$, или, что эквивалентно, матрица вторых производных потенциала (4) должна иметь положительные собственные значения.

Хорошо известен частный случай $\lambda_{6}=\lambda_{7}=0$, соответствующий дискретной симметрии $\mathbb{Z}_{2}$, определяющей инвариантность $U\left(\Phi_{1}, \Phi_{2}\right)$ относительно симметрии Печчеи-Куинн $U_{\mathrm{PQ}}(1)$ [18], нарушение которой приводит к появлению аксиона [19] (ценного с точки зрения возможностей устранения тета-члена, приводящего к нарушению $C P$-инвариантности в $\mathrm{KXД).} \mathrm{В} \mathrm{этом} \mathrm{случае} \mathrm{массовая} \mathrm{матрица} \mathrm{для} \mathrm{полей} \chi_{1}$ и $\chi_{2}$ имеет вид

$$
\left(\begin{array}{cc}
c_{\beta} & s_{\beta} \\
-s_{\beta} & c_{\beta}
\end{array}\right)\left(\begin{array}{cc}
\lambda_{1} v_{1}^{2}+\left(\lambda_{34}-\frac{\lambda_{5}}{2}\right) v_{2}^{2}-\mu_{1}^{2} & -\mu_{12}^{2}+\lambda_{5} v_{1} v_{2} \\
-\mu_{12}^{2}+\lambda_{5} v_{1} v_{2} & \lambda_{2} v_{2}^{2}+\left(\lambda_{34}-\frac{\lambda_{5}}{2}\right) v_{1}^{2}-\mu_{2}^{2}
\end{array}\right)\left(\begin{array}{cc}
c_{\beta} & -s_{\beta} \\
s_{\beta} & c_{\beta}
\end{array}\right) .
$$


Для обеспечения необходимого вида диагонализованной массовой матрицы ${ }^{5)} M_{i j}=$ $\left((0,0),\left(0, M_{22}\right)\right)$ условие для безмассовой моды $M_{11}=0$ (голдстоун) достигается, если выполняется условие

$$
\lambda_{5}=\frac{\mu_{12}^{2}}{v_{1} v_{2}}-\frac{m_{A}^{2}}{2 v^{2}} .
$$

В случае древесного потенциала МCCM имеем $\mu_{12}^{2}=m_{A}^{2} \sin \beta \cos \beta$. Это равенство не выполняется, если ненулевая константа $\lambda_{5}$ генерируется радиационными поправками.

Условия (5), обеспечивающие сокращение линейных по полям членов потенциала (1), можно рассматривать как систему двух уравнений третьей степени по отношению к $v_{1}, v_{2}$, аналитический вид решений которых в общем случае отсутствует. Для расчетов сечений процессов в рамках МССМ обычно сначала фиксируется экстремум эффективного потенциала (1) в полярных координахах $\left(v, \operatorname{arctg} v_{2} / v_{1}\right)$ при заданном значении параметра $\operatorname{tg} \beta$ на окружности $\sqrt{v_{1}^{2}+v_{2}^{2}}=v=246$ ГэВ. Если зафиксировать параметр $m_{A}$, то $\mu_{12}^{2}$ определяется формулой (9), а параметры $\mu_{1}^{2}$, $\mu_{2}^{2}$ задаются в (5), что определяет однозначно эффективный потенциал. Отсутствие аналитических форм в минимуме неудобно для анализа температурной эволюции потенциала, в ходе которой набор $\left(m_{A}(T), \operatorname{tg} \beta(T)\right)$ не фиксирован. Аналитические выражения можно получить путем линейного преобразования, обращающего член $\mu_{12}^{2} v_{1} v_{2}$ в нуль. Для этого нужно провести поворот в пространстве фоновых полей $\left(v_{1}, v_{2}\right)$ :

$$
\left(\begin{array}{l}
v_{1} \\
v_{2}
\end{array}\right)=\left(\begin{array}{cc}
c_{\theta} & -s_{\theta} \\
s_{\theta} & c_{\theta}
\end{array}\right)\left(\begin{array}{l}
\bar{v}_{1} \\
\bar{v}_{2}
\end{array}\right), \quad \operatorname{tg} 2 \theta=\frac{2 \mu_{12}^{2}}{\mu_{1}^{2}-\mu_{2}^{2}},
$$

здесь и далее величины в повернутой системе обозначаются чертой сверху. После поворота $(\operatorname{tg} \beta \rightarrow \operatorname{tg}(\beta-\theta))$ форма потенциала не сохраняется (например, в древесном потенциале МССМ появляются ненулевые $\bar{\lambda}_{6}$ и $\bar{\lambda}_{7}$ ), однако массы пяти скаляров и их константы взаимодействия инвариантны относительно вращения в пространстве фоновых полей ${ }^{6}$. В повернутой системе координат для фоновых полей потенциал примет вид

$$
\bar{U}\left(\bar{v}_{1}, \bar{v}_{2}\right)=-\frac{\bar{\mu}_{1}^{2}}{2} \bar{v}_{1}^{2}-\frac{\bar{\mu}_{2}^{2}}{2} \bar{v}_{2}^{2}+\frac{\bar{\lambda}_{1}}{4} \bar{v}_{1}^{4}+\frac{\bar{\lambda}_{2}}{4} \bar{v}_{2}^{4}+\frac{\bar{\lambda}_{345}}{4} \bar{v}_{1}^{2} \bar{v}_{2}^{2}+\frac{\bar{\lambda}_{6}}{2} \bar{v}_{1}^{3} \bar{v}_{2}+\frac{\bar{\lambda}_{7}}{2} \bar{v}_{1} \bar{v}_{2}^{3},
$$

где переопределенные параметры имеют следующий вид:

$$
\begin{aligned}
& \bar{\mu}_{1,2}^{2}=\frac{\mu_{1}^{2}+\mu_{2}^{2}}{2} \mp \frac{D}{2}, \quad D=\sqrt{\left(\mu_{1}^{2}-\mu_{2}^{2}\right)^{2}+4 \mu_{12}^{4}}, \\
& \bar{\lambda}_{1,2}=\frac{1}{D^{2}}\left[\frac{\lambda_{1}+\lambda_{2}}{2}\left(\mu_{1}^{2}-\mu_{2}^{2}\right)^{2}+\mu_{12}^{4}\left(\lambda_{1}+\lambda_{2}+\lambda_{345}\right)\right] \pm \frac{1}{2 D}\left|\mu_{1}^{2}-\mu_{2}^{2}\right|\left(-\lambda_{1}+\lambda_{2}\right), \\
& \bar{\lambda}_{345}=\frac{1}{D^{2}}\left\{6\left(\lambda_{1}+\lambda_{2}\right) \mu_{12}^{4}+\lambda_{345}\left[\left(\mu_{1}^{2}-\mu_{2}^{2}\right)^{2}-2 \mu_{12}^{4}\right]\right\}, \\
& \bar{\lambda}_{6,7}=-\frac{\mu_{12}^{2}}{D}\left[-\lambda_{1}+\lambda_{2} \pm \frac{\left|\mu_{1}^{2}-\mu_{2}^{2}\right|}{D}\left(\lambda_{1}+\lambda_{2}-\lambda_{345}\right)\right] .
\end{aligned}
$$

${ }^{5)}$ Согласно принятым в литературе обозначениям бозонов Хиггса МССМ-фактор $M_{22}=m_{A}^{2} / 2$ перед членом лагранжиана размерности $2\left(A^{2}\right)$ содержит массу $C P$-нечетного бозона Хиггса $m_{A}$.

${ }^{6)} \mathrm{B}$ частности, параметризация двухдублетного потенциала МССМ с сектором Юкавы типа III при помощи переменной $\operatorname{tg} \beta$ не имеет физического смысла, так как различным значениям $\operatorname{tg} \beta$ соответствует один и тот же спектр масс бозонов Хиггса; см. детальное обсуждение в [20].

6 Теоретическая и математическая физика, т. 184, № 2, 2015 г. 
Большое количество свободных параметров общей двухдублетной модели существенно ограничивается в рамках МССМ.

На масштабе энергии $M_{\mathrm{SUSY}}$ порядка масс суперпартнеров эффективный потенциал $U\left(\Phi_{1}, \Phi_{2}\right)$ приводится к древесному потенциалу MCCM $U_{\text {tree }}\left(\Phi_{1}, \Phi_{2}\right)$, где $\lambda_{1}=\lambda_{2}=\left(g_{1}^{2}+g_{2}^{2}\right) / 8, \lambda_{3}=\left(g_{2}^{2}-g_{1}^{2}\right) / 4, \lambda_{4}=-g_{2}^{2} / 2, \lambda_{5}=\lambda_{6}=\lambda_{7}=0$. Обозначим $\bar{g}^{2}=g_{1}^{2}+g_{2}^{2}, \lambda_{345}=\lambda_{3}+\lambda_{4}+\lambda_{5}=-\bar{g}^{2} / 4$. В дальнейшем для качественных численных оценок будем использовать константы взаимодействия четырех скалярных полей в МССМ на этом масштабе

$$
\begin{aligned}
\bar{\lambda}_{1} & =\bar{\lambda}_{2}=\frac{\bar{g}^{2}}{32} c_{2 \theta}^{2}=\frac{\bar{g}^{2}}{8 D^{2}}\left(\mu_{1}^{2}-\mu_{2}^{2}\right)^{2}, \\
\bar{\lambda}_{345} & =\frac{\bar{g}^{2}}{32}\left(1-3 c_{4 \theta}\right)=\frac{\bar{g}^{2}}{4 D^{2}}\left[-\left(\mu_{1}^{2}-\mu_{2}^{2}\right)^{2}+8 \mu_{12}^{4}\right], \\
\bar{\lambda}_{6} & =-\bar{\lambda}_{7}=\frac{\bar{g}^{2}}{16} s_{4 \theta}=-\frac{\bar{g}^{2}}{2 D^{2}} \mu_{12}^{2}\left|\mu_{1}^{2}-\mu_{2}^{2}\right| .
\end{aligned}
$$

Прямую проверку инвариантности масс бозонов Хиггса $m_{h}, m_{H}, m_{A}, m_{H^{ \pm}}$относительно поворота (10) в пространстве фоновых полей можно выполнить, используя преобразование потенциала (1) в массовый базис [12]

$$
\begin{aligned}
& \lambda_{1}=\frac{1}{2 v^{2}}\left[\left(\frac{s_{\alpha}}{c_{\beta}}\right)^{2} m_{h}^{2}+\left(\frac{c_{\alpha}}{c_{\beta}}\right)^{2} m_{H}^{2}-\frac{s_{\beta}}{c_{\beta}^{3}} \mu_{12}^{2}\right]+\frac{1}{4}\left(\lambda_{7} \operatorname{tg}^{3} \beta-3 \lambda_{6} \operatorname{tg} \beta\right), \\
& \lambda_{2}=\frac{1}{2 v^{2}}\left[\left(\frac{c_{\alpha}}{s_{\beta}}\right)^{2} m_{h}^{2}+\left(\frac{s_{\alpha}}{s_{\beta}}\right)^{2} m_{H}^{2}-\frac{c_{\beta}}{s_{\beta}^{3}} \mu_{12}^{2}\right]+\frac{1}{4}\left(\lambda_{6} \operatorname{ctg}^{3} \beta-3 \lambda_{7} \operatorname{ctg} \beta\right), \\
& \lambda_{3}=\frac{1}{v^{2}}\left[2 m_{H^{ \pm}}^{2}-\frac{\mu_{12}^{2}}{s_{\beta} c_{\beta}}+\frac{s_{2 \alpha}}{s_{2 \beta}}\left(m_{H}^{2}-m_{h}^{2}\right)\right]-\frac{1}{2}\left(\lambda_{6} \operatorname{ctg} \beta+\lambda_{7} \operatorname{tg} \beta\right), \\
& \lambda_{4}=\frac{1}{v^{2}}\left(\frac{\mu_{12}^{2}}{s_{\beta} c_{\beta}}+m_{A}^{2}-2 m_{H^{ \pm}}^{2}\right)-\frac{1}{2}\left(\lambda_{6} \operatorname{ctg} \beta+\lambda_{7} \operatorname{tg} \beta\right), \\
& \lambda_{5}=\frac{1}{v^{2}}\left(\frac{\mu_{12}^{2}}{s_{\beta} c_{\beta}}-m_{A}^{2}\right)-\frac{1}{2}\left(\lambda_{6} \operatorname{ctg} \beta+\lambda_{7} \operatorname{tg} \beta\right),
\end{aligned}
$$

откуда получаем массы $C P$-четных скаляров

$$
\begin{gathered}
m_{h}^{2}=c_{\alpha-\beta}^{2} m_{A}^{2}+v^{2}\left[2 \lambda_{1} s_{\alpha}^{2} c_{\beta}^{2}+2 \lambda_{2} c_{\alpha}^{2} s_{\beta}^{2}-2\left(\lambda_{3}+\lambda_{4}\right) c_{\alpha} c_{\beta} s_{\alpha} s_{\beta}+\right. \\
\left.\quad+\lambda_{5}\left(s_{\alpha}^{2} s_{\beta}^{2}+c_{\alpha}^{2} c_{\beta}^{2}\right)-2 c_{\alpha+\beta}\left(\lambda_{6} s_{\alpha} c_{\beta}-\lambda_{7} c_{\alpha} s_{\beta}\right)\right] \\
m_{H}^{2}=s_{\alpha-\beta}^{2} m_{A}^{2}+v^{2}\left[2 \lambda_{1} c_{\alpha}^{2} c_{\beta}^{2}+2 \lambda_{2} s_{\alpha}^{2} s_{\beta}^{2}+2\left(\lambda_{3}+\lambda_{4}\right) c_{\alpha} c_{\beta} s_{\alpha} s_{\beta}+\right. \\
\left.\quad+\lambda_{5}\left(c_{\alpha}^{2} s_{\beta}^{2}+s_{\alpha}^{2} c_{\beta}^{2}\right)+2 s_{\alpha+\beta}\left(\lambda_{6} c_{\alpha} c_{\beta}+\lambda_{7} s_{\alpha} s_{\beta}\right)\right]
\end{gathered}
$$

и угол смешивания $\alpha$,

$$
\operatorname{tg} 2 \alpha=\frac{s_{2 \beta} m_{A}^{2}-v^{2}\left(\left(\lambda_{3}+\lambda_{4}\right) s_{2 \beta}+2 c_{\beta}^{2} \lambda_{6}+2 s_{\beta}^{2} \lambda_{7}\right)}{c_{2 \beta} m_{A}^{2}-v^{2}\left(2 \lambda_{1} c_{\beta}^{2}-2 \lambda_{2} s_{\beta}^{2}-\lambda_{5} c_{2 \beta}+\left(\lambda_{6}-\lambda_{7}\right) s_{2 \beta}\right)} .
$$

Масса псевдоскаляра связана с массой заряженного бозона соотношением

$$
m_{H^{ \pm}}^{2}=m_{A}^{2}\left(1+\frac{g_{2}^{2}}{\bar{g}^{2}} \frac{s_{2(\alpha-\beta)}}{s_{2(\alpha+\beta)}}\right) .
$$


Переопределяя $\operatorname{tg} \beta \rightarrow \operatorname{tg}(\beta-\theta)$ и используя соотношения (12) для перехода от $\lambda_{i}$ к $\bar{\lambda}_{i}$, легко проверить не следующую очевидно из выражений (15), (16) инвариантность масс бозонов относительно преобразования (10) фоновых полей.

\section{3. ПРИВЕДЕНИЕ ЭФФЕКТИВНОГО ПОТЕНЦИАЛА К КАНОНИЧЕСКОЙ ФОРМЕ МОРСА}

В данной работе исследуется эффективный потенциал Хиггса МССМ при конечных температурах [15], построение которого основано на расчете различных однопетлевых температурных поправок, возникающих в секторе МСCM, который определяет взаимодействие третьего поколения суперпартнеров кварков с хиггсовскими изодублетами.

В силу температурной эволюции потенциала Хиггса его вакуумные конденсаты $v_{1}(T), v_{2}(T)$ также меняют свои значения: в симметричной фазе при $T>100$ ГэВ они равны $v_{1,2}=0$, в нарушенной фазе при $T=0$ наблюдаемая в настоящее время плотность энергии вакуума $v=\sqrt{v_{1}^{2}+v_{2}^{2}}=246$ ГэВ. Большой интерес представляет вопрос исследования их промежуточных значений.

В случае равновесной термодинамической эволюции, когда возможна интерпретация системы в терминах скалярных состояний $h, H, A, H^{ \pm}$, значения вакуумных конденсатов меняются плавно и непрерывно. Согласно [15] при некоторой критической температуре $T_{\mathrm{c}} \sim 100$ ГэВ потенциал Хиггса в пространстве $\left(v_{1}, v_{2}\right)$ имеет седлообразную форму и, соответственно, неустойчивый минимум. При температуре

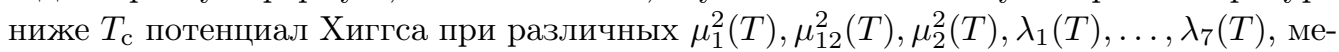
няя свою форму, сохраняет минимум устойчивым. Последний может перемещаться в пространстве $\left(v_{1}, v_{2}\right)$ как по прямой линии (см. [15]), так и по некоторой кривой.

Однако система может не всегда находиться в состоянии термодинамического равновесия. Если в ранней Вселенной имел место электрослабый фазовый переход первого рода, вакуумные конденсаты могли менять свои значения скачком при критической температуре $T_{c}$. Описанная выше картина поведения $\left(v_{1}, v_{2}\right)$ в этом случае может быть неадекватна фазовому переходу первого рода и для обеспечения скачков имеет смысл пересмотреть методы анализа. Наглядное описание эволюции системы в этом случае дает теория катастроф, в рамках которой исследуется многообразие катастрофы в пространстве переменных состояния системы и математических управляющих параметров.

В рамках теории катастроф имеются отработанные методы анализа функций вблизи их критических точек. Если определитель матрицы устойчивости $\operatorname{det} U_{i j}=$ $\operatorname{det} \partial^{2} U / \partial v_{i} \partial v_{j}$ не равен нулю, то лемма Морса [16] гарантирует существование гладкой нелинейной замены переменных в окрестности критической точки $v_{1}, v_{2} \rightarrow \bar{v}_{1}^{\prime}, \bar{v}_{2}^{\prime}$ такой, что функция $U$ может быть представлена в канонической форме $U=\Sigma_{i} \Lambda_{i}\left(\bar{v}_{i}^{\prime}\right)^{2}$, где $\Lambda_{i}-$ собственные значения матрицы устойчивости в стационарной точке (морсовское $i$-седло, $\left.M_{i}\right)$. Если $\operatorname{det} U_{i j}=0(l$ собственных значений матрицы устойчивости равны нулю), то функция $U$ может быть представлена в виде суммы формы Морса и некоторой гладкой функции $f_{\mathrm{NM}}$, зависящей от $l$ переменных (неморсовской части): $U=f_{\mathrm{NM}}+M_{i}^{n-l}$, где $\partial^{2} f / \partial v_{i} \partial v_{j}=0, \partial^{2} M / \partial v_{i} \partial v_{j} \neq 0$, причем теорема Тома [16] при количестве управляющих параметров менее пяти гарантирует существование гладкой нелинейной замены переменных такой, что функция $U$ может 
ТАБлицА 1. Условия локального минимума для четырех типов решений системы (17).

\begin{tabular}{|c|c|c|c|}
\hline & Решение (17) & Гессиан & Условия минимума \\
\hline 1 & $\bar{v}_{1}=0, \bar{v}_{2}=0$ & $-\left(\begin{array}{cc}\bar{\mu}_{1}^{2} & 0 \\
0 & \bar{\mu}_{2}^{2}\end{array}\right)$ & $\bar{\mu}_{1}^{2}+\bar{\mu}_{2}^{2}<0, \quad \bar{\mu}_{1}^{2} \bar{\mu}_{2}^{2} \geqslant 0$ \\
\hline 2 & $\bar{v}_{1}=0, \bar{\lambda}_{2} \bar{v}_{2}^{2}-\bar{\mu}_{2}^{2}=0$ & $\left(\begin{array}{cc}-\bar{\mu}_{1}^{2}+\frac{1}{2} \bar{\lambda}_{345} \bar{v}_{2}^{2} & 0 \\
0 & 2{\overline{\lambda_{2}}}^{2} \bar{v}_{2}^{2}\end{array}\right)$ & $\begin{array}{c}-\bar{\mu}_{1}^{2}+\bar{v}_{2}^{2}\left(2 \bar{\lambda}_{2}+\frac{1}{2} \bar{\lambda}_{345}\right)>0 \\
\left(-\bar{\mu}_{1}^{2}+\frac{1}{2} \bar{\lambda}_{345} \bar{v}_{2}^{2}\right) \bar{\lambda}_{2} \bar{v}_{2}^{2} \geqslant 0\end{array}$ \\
\hline 3 & $\bar{v}_{2}=0, \bar{\lambda}_{1} \bar{v}_{1}^{2}-\bar{\mu}_{1}^{2}=0$ & $\left(\begin{array}{cc}2 \bar{\lambda}_{1} \bar{v}_{1}^{2} & 0 \\
0 & -\bar{\mu}_{2}^{2}+\frac{1}{2} \bar{\lambda}_{345} \bar{v}_{1}^{2}\end{array}\right)$ & $\begin{array}{c}-\bar{\mu}_{2}^{2}+\bar{v}_{1}^{2}\left(2 \bar{\lambda}_{1}+\frac{1}{2} \bar{\lambda}_{345}\right)>0 \\
\left(-\bar{\mu}_{2}^{2}+\frac{1}{2} \bar{\lambda}_{345} \bar{v}_{1}^{2}\right) \bar{\lambda}_{1} \bar{v}_{1}^{2} \geqslant 0\end{array}$ \\
\hline 4 & $\begin{array}{l}\bar{\lambda}_{1} \bar{v}_{1}^{2}+\frac{1}{2} \bar{\lambda}_{345} \bar{v}_{2}^{2}-\bar{\mu}_{1}^{2}=0, \\
\bar{\lambda}_{2} \bar{v}_{2}^{2}+\frac{1}{2} \bar{\lambda}_{345} \bar{v}_{1}^{2}-\bar{\mu}_{2}^{2}=0\end{array}$ & $\left(\begin{array}{cc}2 \bar{\lambda}_{1} \bar{v}_{1}^{2} & \bar{\lambda}_{345} \bar{v}_{1} \bar{v}_{2} \\
\bar{\lambda}_{345} \bar{v}_{1} \bar{v}_{2} & 2 \bar{\lambda}_{2} \bar{v}_{2}^{2}\end{array}\right)$ & $\begin{array}{c}\bar{\lambda}_{1} \bar{v}_{1}^{2}+\bar{\lambda}_{2} \bar{v}_{2}^{2}>0, \\
\bar{v}_{1}^{2} \bar{v}_{2}^{2}\left(4 \bar{\lambda}_{1} \bar{\lambda}_{2}-\bar{\lambda}_{345}^{2}\right) \geqslant 0\end{array}$ \\
\hline
\end{tabular}

быть записана в канонической форме

$$
U=C G(l)+\sum_{j=l+1}^{n} \Lambda_{j} v_{j}^{2},
$$

где $C G(l)$ представляет собой росток катастрофы.

Выше упоминалось, что в общем случае аналитические решения неоднородной системы уравнений третьей степени (5) для экстремума потенциала отсутствуют. Отметим, что в специальном случае $\bar{\lambda}_{6}=\bar{\lambda}_{7}=0$ после поворота (10) эти уравнения факторизуются,

$$
\begin{aligned}
& \bar{v}_{1}\left(\bar{\lambda}_{1} \bar{v}_{1}^{2}+\frac{\bar{\lambda}_{345}}{2} \bar{v}_{2}^{2}-\bar{\mu}_{1}^{2}\right)=0 \\
& \bar{v}_{2}\left(\bar{\lambda}_{2} \bar{v}_{2}^{2}+\frac{\bar{\lambda}_{345}}{2} \bar{v}_{2}^{2}-\bar{\mu}_{2}^{2}\right)=0
\end{aligned}
$$

определяя четыре набора критических точек. Соответствующие матрицы вторых производных и условия минимума [15] приведены в табл. 1.

Для наиболее простого случая термодинамической эволюции вакуумных средних $v_{1}(T), v_{2}(T)$ параметр $\operatorname{tg} \beta$ не зависит от температуры. Тогда эффективный потенциал (4) принимает вид

$$
U=-\mu^{2} v^{2}+\lambda v^{4}
$$


где

$$
\begin{aligned}
\lambda & =\frac{c_{\beta}^{8}}{64}\left(\lambda_{2} \operatorname{tg}^{4} \beta+2 \lambda_{7} \operatorname{tg}^{3} \beta+\lambda_{345} \operatorname{tg}^{2} \beta+2 \lambda_{6} \operatorname{tg} \beta+\lambda_{1}\right), \\
\mu^{2} & =\frac{c_{\beta}^{4}}{8}\left(\mu_{2}^{2} \operatorname{tg}^{2} \beta+2 \mu_{12}^{2} \operatorname{tg} \beta+\mu_{1}^{2}\right),
\end{aligned}
$$

параметры $\lambda_{i}=\lambda_{i}(T), i=1, \ldots, 7, \mu_{1,2,12}=\mu_{1,2,12}(T)$.

Имеем две критические точки потенциала (18): $v^{(1)}=0$ и $v^{(2)}= \pm \sqrt{\alpha}$, где $\alpha=\mu^{2} / 2 \lambda$. Первая критическая точка соответствует симметричной фазе, вторая (при $\alpha>0$ ) соответствует двум минимумам. Определитель матрицы устойчивости в стационарных точках $\left.\operatorname{det} D^{2} U\right|_{v^{(1)}}=-2 \mu^{2},\left.\operatorname{det} D^{2} U\right|_{v^{(2)}}=4 \mu^{2}$, каноническая форма потенциала (или морсовская форма) $U=\lambda\left(\bar{v}^{2}-\alpha^{2}\right)$, где $\bar{v}=v^{2}-\alpha$. В системе не происходит бифуркаций.

Сложнее представляется случай $v_{2}(T) / v_{1}(T)=\operatorname{tg} \beta(T)$, когда $v_{1}(T), v_{2}(T)$ независимы и потенциал (4) имеет критическую точку, определяемую условиями (5). Собственные значения матрицы устойчивости $U_{i j}$ в этом случае имеют вид

$$
2 \Lambda_{1,2}=-\frac{1}{2}\left(\mu_{1}^{2}+\mu_{2}^{2}\right) \pm \frac{1}{2} D
$$

или $\Lambda_{1,2}=-\bar{\mu}_{1,2}^{2} / 2$, где $\bar{\mu}_{1,2}^{2}=\left(\mu_{1}^{2}+\mu_{2}^{2}\right) / 2 \mp D / 2$.

Рассмотрим случай $\operatorname{det} U_{i j} \neq 0$ и приведем потенциал (4) к канонической форме ${ }^{7}$

$$
\bar{U}^{\prime}\left(\bar{v}_{1}^{\prime}, \bar{v}_{2}^{\prime}\right)=-\frac{\bar{\mu}_{1}^{2}}{2} \bar{v}_{1}^{\prime 2}-\frac{\bar{\mu}_{2}^{2}}{2} \bar{v}_{2}^{\prime 2} .
$$

Для этого преобразуем потенциал в общем базисе (4) к виду (11), исключив смешанный член размерности 2 по полям, после чего выполним нелинейное осесохраняющее преобразование

$$
\begin{aligned}
& \bar{v}_{1}^{\prime}=\bar{v}_{1}+\left(A_{20} \bar{v}_{1}^{2}+A_{11} \bar{v}_{1} \bar{v}_{2}+A_{02} \bar{v}_{2}^{2}\right)+\left(A_{30} \bar{v}_{1}^{3}+A_{21} \bar{v}_{1}^{2} \bar{v}_{2}+A_{12} \bar{v}_{1} \bar{v}_{2}^{2}+A_{03} \bar{v}_{2}^{3}\right), \\
& \bar{v}_{2}^{\prime}=\bar{v}_{2}+\left(B_{20} \bar{v}_{1}^{2}+B_{11} \bar{v}_{1} \bar{v}_{2}+B_{02} \bar{v}_{2}^{2}\right)+\left(B_{30} \bar{v}_{1}^{3}+B_{21} \bar{v}_{1}^{2} \bar{v}_{2}+B_{12} \bar{v}_{1} \bar{v}_{2}^{2}+B_{03} \bar{v}_{2}^{3}\right) .
\end{aligned}
$$

Ограничимся членами не выше третьей степени по вакуумным конденсатам, что дает форму (21) с точностью до членов шестой степени. После подстановки (22) в (21) получим представленную в табл. 2 систему уравнений для определения $A_{i j}$ и $B_{i j}$. Сразу видно, что $A_{20}=B_{02}=0$, и система уравнений совместна в следующих девяти случаях:
1) $A_{02}=A_{03}=B_{20}=B_{30}=0$;
4) $A_{02}=A_{03}=B_{20}=0$;
7) $B_{20}=A_{03}=0$;
2) $B_{20}=B_{30}=A_{02}=0$;
5) $A_{02}=A_{03}=B_{30}=0$;
8) $B_{30}=A_{02}=0$;
3) $B_{20}=B_{30}=A_{03}=0$;
6) $B_{20}=A_{02}=0$;
9) $B_{30}=A_{03}=0$.

Поэтому возможны пять аналитических форм нелинейных преобразований (22) без дополнительных условий для управляющих параметров и пять форм с дополнительными условиями связи между управляющими параметрами. Подробные выкладки для девяти случаев можно найти в работе [21]. Члены шестой степени образуют три

7)Заметим, что по определению канонической морсовской формой является переопределенная форма $U=-\tilde{v}_{1}^{2}-\tilde{v}_{2}^{2}$, где $\tilde{v}_{i}=\left(\bar{\mu}_{i} / \sqrt{2}\right) \bar{v}_{i}^{\prime}$. 
ТАБлица 2. Условия, определяющие факторы $A_{i j}$ и $B_{i j}$ в нелинейных преобразованиях (22), приводящих потенциал (4) к канонической форме.

\begin{tabular}{|c|c|}
\hline Поля $\bar{v}_{1}^{i} \bar{v}_{2}^{j}$ & $C_{i j}-$ факторы при $\bar{v}_{i} \bar{v}_{j}$ в $\bar{U}\left(\bar{v}_{1}, \bar{v}_{2}\right)$ \\
\hline $\bar{v}_{1}^{3}$ & $A_{20} \bar{\mu}_{1}^{2}=0$ \\
\hline $\bar{v}_{1} \bar{v}_{2}^{2}$ & $A_{02} \bar{\mu}_{1}^{2}+B_{11} \bar{\mu}_{2}^{2}=0$ \\
\hline $\bar{v}_{1}^{2} \bar{v}_{2}$ & $A_{11} \bar{\mu}_{1}^{2}+B_{20} \bar{\mu}_{2}^{2}=0$ \\
\hline $\bar{v}_{2}^{3}$ & $B_{02} \bar{\mu}_{2}^{2}=0$ \\
\hline $\bar{v}_{1}^{4}$ & $-\frac{1}{2} A_{20}^{2} \bar{\mu}_{1}^{2}-A_{30} \bar{\mu}_{1}^{2}-\frac{1}{2} B_{20}^{2} \bar{\mu}_{2}^{2}=\frac{\bar{\lambda}_{1}}{4}$ \\
\hline $\bar{v}_{1} \bar{v}_{2}^{3}$ & $-\left(A_{03}+A_{02} A_{11}\right) \bar{\mu}_{1}^{2}-\left(B_{02} B_{11}+B_{12}\right) \bar{\mu}_{2}^{2}=\frac{\bar{\lambda}_{7}}{2}$ \\
\hline $\bar{v}_{1}^{2} \bar{v}_{2}^{2}$ & $-\left(A_{11}^{2}+2\left(A_{12}+A_{02} A_{20}\right)\right) \bar{\mu}_{1}^{2}-\left(B_{11}^{2}+2\left(B_{21}+B_{02} B_{20}\right)\right) \bar{\mu}_{2}^{2}=\frac{\bar{\lambda}_{345}}{2}$ \\
\hline $\bar{v}_{1}^{3} \bar{v}_{2}$ & $-\left(A_{21}+A_{11} A_{20}\right) \bar{\mu}_{1}^{2}-\left(B_{11} B_{20}+B_{30}\right) \bar{\mu}_{2}^{2}=\frac{\bar{\lambda}_{6}}{2}$ \\
\hline $\bar{v}_{2}^{4}$ & $-\frac{1}{2} A_{02}^{2} \bar{\mu}_{1}^{2}-B_{03} \bar{\mu}_{2}^{2}-\frac{1}{2} B_{02}^{2} \bar{\mu}_{2}^{2}=\frac{\bar{\lambda}_{2}}{4}$ \\
\hline $\bar{v}_{1}^{5}$ & $A_{20} A_{30} \bar{\mu}_{1}^{2}+B_{20} B_{30} \bar{\mu}_{2}^{2}=0$ \\
\hline $\bar{v}_{1} \bar{v}_{2}^{4}$ & $\left(A_{03} A_{11}+A_{02} A_{12}\right) \bar{\mu}_{1}^{2}+\left(B_{03} B_{11}+B_{02} B_{12}\right) \bar{\mu}_{2}^{2}=0$ \\
\hline $\bar{v}_{1}^{2} \bar{v}_{2}^{3}$ & $\left(A_{11} A_{12}+A_{03} A_{20}+A_{02} A_{21}\right) \bar{\mu}_{1}^{2}+\left(B_{11} B_{12}+B_{03} B_{20}+B_{02} B_{21}\right) \bar{\mu}_{2}^{2}=0$ \\
\hline $\bar{v}_{1}^{3} \bar{v}_{2}^{2}$ & $\left(A_{12} A_{20}+A_{11} A_{21}+A_{02} A_{30}\right) \bar{\mu}_{1}^{2}+\left(B_{12} B_{20}+B_{11} B_{21}+B_{02} B_{30}\right) \bar{\mu}_{2}^{2}=0$ \\
\hline $\bar{v}_{1}^{4} \bar{v}_{2}$ & $\left(A_{20} A_{21}+A_{11} A_{30}\right) \bar{\mu}_{1}^{2}+\left(B_{20} B_{21}+B_{11} B_{30}\right) \bar{\mu}_{2}^{2}=0$ \\
\hline $\bar{v}_{2}^{5}$ & $A_{02} A_{03} \bar{\mu}_{1}^{2}+B_{02} B_{03} \bar{\mu}_{2}^{2}=0$ \\
\hline $\bar{v}_{1}^{6}$ & $A_{30}^{2} \bar{\mu}_{1}^{2}+B_{30}^{2} \bar{\mu}_{2}^{2}$ \\
\hline $\bar{v}_{1} \bar{v}_{2}^{5}$ & $A_{03} A_{12} \bar{\mu}_{1}^{2}+B_{03} B_{12} \bar{\mu}_{2}^{2}$ \\
\hline $\bar{v}_{1}^{2} \bar{v}_{2}^{4}$ & $\left(A_{12}^{2}+2 A_{03} A_{21}\right) \bar{\mu}_{1}^{2}+\left(B_{12}^{2}+2 B_{03} B_{21}\right) \bar{\mu}_{2}^{2}$ \\
\hline $\bar{v}_{1}^{3} \bar{v}_{2}^{3}$ & $\left(A_{12} A_{21}+A_{03} A_{30}\right) \bar{\mu}_{1}^{2}+\left(B_{12} B_{21}+B_{03} B_{30}\right) \bar{\mu}_{2}^{2}$ \\
\hline $\bar{v}_{1}^{4} \bar{v}_{2}^{2}$ & $\left(A_{21}^{2}+2 A_{12} A_{30}\right) \bar{\mu}_{1}^{2}+\left(B_{21}^{2}+2 B_{12} B_{30}\right) \bar{\mu}_{2}^{2}$ \\
\hline $\bar{v}_{1}^{5} \bar{v}_{2}$ & $A_{21} A_{30} \bar{\mu}_{1}^{2}+B_{21} B_{30} \bar{\mu}_{2}^{2}$ \\
\hline $\bar{v}_{2}^{6}$ & $A_{03}^{2} \bar{\mu}_{1}^{2}+B_{03}^{2} \bar{\mu}_{2}^{2}$ \\
\hline
\end{tabular}

набора, внутри каждого из которых для разных форм нелинейных преобразований члены шестой степени совпадают. Например, для случая 1 нелинейное преобразование вакуумных конденсатов имеет вид

$$
\begin{aligned}
& \bar{v}_{1}^{\prime}=\bar{v}_{1}-\frac{\bar{\lambda}_{1}}{4 \bar{\mu}_{1}^{2}} \bar{v}_{1}^{3}-\frac{\bar{\lambda}_{6}}{2 \bar{\mu}_{1}^{2}} \bar{v}_{1}^{2} \bar{v}_{2}-\frac{\bar{\lambda}_{7} \bar{\lambda}_{345}}{4\left(\bar{\lambda}_{7} \bar{\mu}_{1}^{2}-\bar{\lambda}_{6} \bar{\mu}_{2}^{2}\right)} \bar{v}_{1} \bar{v}_{2}^{2}, \\
& \bar{v}_{2}^{\prime}=\bar{v}_{2}+\frac{\bar{\lambda}_{6} \bar{\lambda}_{345}}{4\left(\bar{\lambda}_{7} \bar{\mu}_{1}^{2}-\bar{\lambda}_{6} \bar{\mu}_{2}^{2}\right)} \bar{v}_{1}^{2} \bar{v}_{2}-\frac{\bar{\lambda}_{7}}{2 \bar{\mu}_{2}^{2}} \bar{v}_{1} \bar{v}_{2}^{2}-\frac{\bar{\lambda}_{2}}{4 \bar{\mu}_{2}^{2}} \bar{v}_{2}^{3}
\end{aligned}
$$

и содержит неудобный полюс при $\bar{\lambda}_{6} \bar{\mu}_{2}^{2}=\bar{\lambda}_{7} \bar{\mu}_{1}^{2}$, проявляющийся в пространстве параметров модели. Наилучшую точность и наиболее простой вид имеет преобра- 


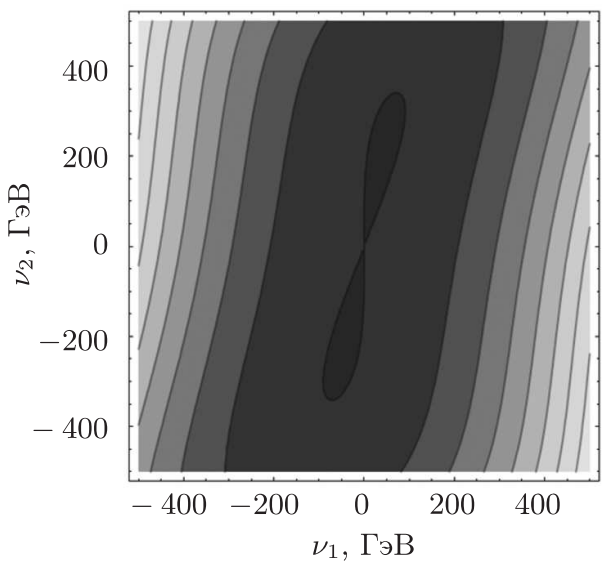

a

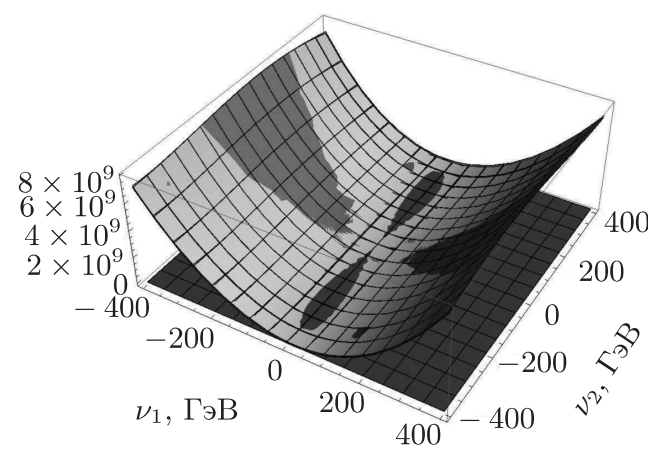

B
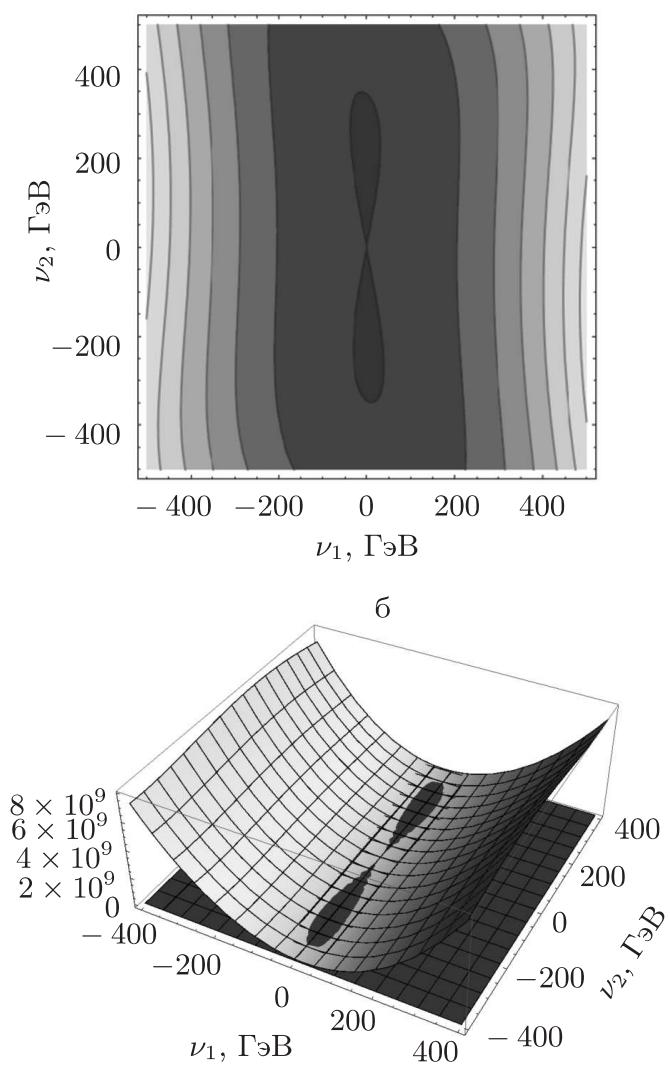

$\Gamma$

Рис. 1. Контуры, соответствующие сечениям потенциалов (4) и (11) плоскостями, параллельными горизонтальной плоскости, до (а) и после (б) поворота на масштабе $M_{\mathrm{SUSY}}$. Сравнение экстремальных поверхностей потенциалов (11) и (21) при учете (в) и без учета (г) членов шестой степени. Члены шестой степени ухудшают точность соответствия поверхностей. Здесь $g_{1}=0.3573, g_{2}=0.6517, m_{A}=300$ ГэВ, $v=246$ ГэВ, $\operatorname{tg} \beta=5$.

зование в случае 6 :

$$
\begin{aligned}
& \bar{v}_{1}^{\prime}=\bar{v}_{1}-\frac{\bar{\lambda}_{1}}{4 \bar{\mu}_{1}^{2}} \bar{v}_{1}^{3}-\frac{\bar{\lambda}_{6}}{2 \bar{\mu}_{1}^{2}} \bar{v}_{1}^{2} \bar{v}_{2}-\frac{\bar{\lambda}_{345}}{4 \bar{\mu}_{1}^{2}} \bar{v}_{1} \bar{v}_{2}^{2}-\frac{\bar{\lambda}_{7}}{2 \bar{\mu}_{1}^{2}} \bar{v}_{2}^{3}, \\
& \bar{v}_{2}^{\prime}=\bar{v}_{2}-\frac{\bar{\lambda}_{2}}{4 \bar{\mu}_{2}^{2}} \bar{v}_{2}^{3} .
\end{aligned}
$$

Ненулевые члены шестой степени принимают вид

$$
\begin{aligned}
& C_{60}=-\frac{\bar{\lambda}_{1}^{2}}{32 \bar{\mu}_{1}^{2}}, \quad C_{15}=-\frac{\bar{\lambda}_{7} \bar{\lambda}_{345}}{8 \bar{\mu}_{1}^{2}}, \quad C_{51}=-\frac{\bar{\lambda}_{1} \bar{\lambda}_{6}}{8 \bar{\mu}_{1}^{2}}, \quad C_{06}=-\frac{\bar{\lambda}_{7}^{2}}{8 \bar{\mu}_{1}^{2}}-\frac{\bar{\lambda}_{2}^{2}}{32 \bar{\mu}_{2}^{2}}, \\
& C_{24}=-\frac{8 \bar{\lambda}_{6} \bar{\lambda}_{7}+\bar{\lambda}_{345}^{2}}{32 \bar{\mu}_{1}^{2}}, \quad C_{33}=-\frac{\bar{\lambda}_{1} \bar{\lambda}_{7}+\bar{\lambda}_{6} \bar{\lambda}_{345}}{8 \bar{\mu}_{1}^{2}}, \quad C_{42}=-\frac{2 \bar{\lambda}_{6}^{2}+\bar{\lambda}_{1} \bar{\lambda}_{345}}{16 \bar{\mu}_{1}^{2}} .
\end{aligned}
$$


Точность приближения потенциала (4) при помощи канонической формы (11) иллюстрируется на рис. 1. Поверхности на рис. 1 а и рис. 16 точно совмещаются после поворота на угол $\theta$; приближение для поверхности (11) при помощи (21) имеет расхождение (по оси $z$ ) порядка $10^{-13} \Gamma_{\ni} \mathrm{B}^{4}$ при $v_{1}$ и $v_{2}$ порядка 0.1 ГэВ, $10^{-8}$ ГэВ ${ }^{4}$ при $v_{1}$ и $v_{2}$ порядка 1 ГэВ; при удалении от начала координат на 10 ГэВ точность ухудшается на шесть порядков до $10^{-2}$ Гэ ${ }^{4}$. Трехмерные изображения поверхностей стационарных точек для других вариантов нелинейных преобразований $\left(\bar{v}_{1}^{\prime}, \bar{v}_{2}^{\prime}\right)$ приведены в работе [21]; другие варианты могут иметь существенно (на порядок) худшую точность.

\section{4. ПРИВЕДЕНИЕ ЭФФЕКТИВНОГО ПОТЕНЦИАЛА К КАНОНИЧЕСКОЙ ФОРМЕ ТОМА}

Рассмотрим случай $\operatorname{det} U_{i j}=0$, когда применима лемма расщепления, в соответствии с которой можно найти росток катастрофы. Возмущение управляющих параметров $\lambda_{i}$ дает функцию-катастрофу. Как уже упоминалось, согласно лемме расщепления критическая точка $v=0$ является вырожденной (или неизолированной), если

$$
\mu_{1}^{2} \mu_{2}^{2}=\mu_{12}^{4} .
$$

В этом случае для формы (7) поверхности стационарных точек $\operatorname{det}\left\|U_{i j}^{\mu}\right\|=0$. Тогда собственные значения (20), полученные ранее, принимают вид

$$
\Lambda_{1}=0, \quad \Lambda_{2}=-\frac{\mu_{1}^{2}+\mu_{2}^{2}}{2} .
$$

Согласно теореме Тома каноническое разложение потенциала Хиггса в открытой окрестности неизолированной критической точки $\left(v=0 ; \lambda_{i}\right)$ имеет вид

$$
U=\Lambda_{2}(T) \bar{v}_{2}^{\prime 2}+\operatorname{Cat}(l, k),
$$

где первое слагаемое - морсовская часть, а второе - функция-катастрофа, определяемая количеством нулевых собственных значений матрицы вторых производных потенциала $l$ и числом управляющих параметров $k$. В рассматриваемом случае $l=1$ (одно собственное значение равно нулю). Число управляющих параметров (т. е. параметров, от которых зависит в общем случае выполнение условия равенства нулю градиента потенциала), как видно из уравнений (5) и условия существования безмассовой голдстоуновской моды, зависит от выбранной модели хиггсовского сектора. В общем случае имеются четыре зависящих от температуры управляющих параметpa $\left(\lambda_{1}, \lambda_{2}, \lambda_{34}, \lambda_{5}\right)$, возмущение которых определяет функцию-катастрофу Cat $(1,4)$, для которой согласно универсальной ADE-классификации [16] принято обозначение катастрофа muna $A_{5}$. В рамках MCCM на масштабе масс суперпартнеров с несущественными квантовыми поправками $\lambda_{1} \sim \lambda_{2}, \lambda_{5}=0$ имеются два управляющих параметра. Тогда неморсовская часть Cat $(1,2)$ классифицируется как катастрофа типа $A_{3}$. Рассмотрим сначала этот случай. Осуществив нелинейное осесохраняющее преобразование

$$
\begin{aligned}
& \bar{v}_{1}^{\prime}=\bar{v}_{1}-\frac{1}{2}\left(1+\frac{\bar{\lambda}_{6}}{2}\right) \bar{v}_{1}^{2}+\frac{5 \bar{\mu}_{2}^{2}\left(2+\bar{\lambda}_{6}\right)^{3}}{16\left(2-4 \bar{\mu}_{2}^{2}+\bar{\lambda}_{1} \bar{\mu}_{2}^{2}\right)} \bar{v}_{1}^{2} \bar{v}_{2}+\frac{1}{2}\left(1+\frac{\bar{\lambda}_{6}}{2}\right)^{2} \bar{v}_{1}^{3}, \\
& \bar{v}_{2}^{\prime}=\bar{v}_{2}+\frac{1}{\bar{\mu}_{2}^{2}} \bar{v}_{1}^{2}-\frac{\bar{\lambda}_{345}}{4 \bar{\mu}_{2}^{2}} \bar{v}_{1}^{2}-\frac{\bar{\lambda}_{7}}{2 \bar{\mu}_{2}^{2}} \bar{v}_{1} \bar{v}_{2}^{2}-\frac{\bar{\lambda}_{2}}{4 \bar{\mu}_{2}^{2}} \bar{v}_{2}^{3},
\end{aligned}
$$


получим каноническую форму Тома, включающую функцию-катастрофу Cat $(1,2)=$ $\bar{v}_{1}^{\prime 4}+a_{1} \bar{v}_{1}^{\prime}+a_{2} \bar{v}_{1}^{\prime 2}[16]$, так что потенциал имеет вид

$$
\bar{U}\left(\bar{v}_{1}^{\prime}, \bar{v}_{2}^{\prime}\right)=\Lambda_{2} \bar{v}_{2}^{\prime 2}+\bar{v}_{1}^{\prime 4}+a_{1} \bar{v}_{1}^{\prime}+a_{2} \bar{v}_{1}^{\prime 2},
$$

где коэффициенты возмущения определяются выражениями

$$
a_{1}=\frac{16\left(2-4 \bar{\mu}_{2}^{2}+\bar{\lambda}_{1} \bar{\mu}_{2}^{2}\right)}{5\left(2+\bar{\lambda}_{6}\right)^{3} \bar{\mu}_{2}^{2}}, \quad a_{2}=\frac{a_{1}}{2}\left(1+\frac{\bar{\lambda}_{6}}{2}\right) .
$$

В более сложном случае существенных квантовых поправок в древесном потенциале МССМ имеются четыре управляющих параметра. Фазовый переход описывается функцией-катастрофой $\operatorname{Cat}(1,4)[16]$, так что потенциал включает функцию-катастрофу типа $A_{5}$

$$
\bar{U}\left(\bar{v}_{1}^{\prime}, \bar{v}_{2}^{\prime}\right)=\Lambda_{2} \bar{v}_{2}^{\prime 2}+\bar{v}_{1}^{\prime 6}+a_{1} \bar{v}_{1}^{\prime}+a_{2} \bar{v}_{1}^{\prime 2}+a_{3} \bar{v}_{1}^{\prime 3}+a_{4} \bar{v}_{1}^{\prime 4},
$$

преобразование к которому с точностью до членов $O\left(\bar{v}_{i}^{7}\right)$ имеет вид

$$
\begin{aligned}
& \bar{v}_{1}^{\prime}=\bar{v}_{1}+\frac{13 \sqrt{6}}{\sqrt{-\bar{\lambda}_{1}^{5}}}\left(\frac{1}{13} \bar{\lambda}_{1}^{2} \bar{v}_{1}^{2}-\frac{8 \sqrt{-\bar{\lambda}_{1}^{3}}}{13 \sqrt{6}} \bar{v}_{1}^{3}+2 \bar{\lambda}_{6} \bar{v}_{1}^{2} \bar{v}_{2}+\bar{\lambda}_{345} \bar{v}_{1}^{2} \bar{v}_{2}^{2}+2 \bar{\lambda}_{7} \bar{v}_{1} \bar{v}_{2}^{3}+\bar{\lambda}_{2} \bar{v}_{2}^{4}\right), \\
& \bar{v}_{2}^{\prime}=\bar{v}_{2}+\sqrt{\frac{3}{2} \frac{1}{\sqrt{-\bar{\lambda}_{1}}} \bar{\mu}_{2}^{2}}\left(2 \bar{\lambda}_{6} \bar{v}_{1}^{4}+\bar{\lambda}_{345} \bar{v}_{1}^{3} \bar{v}_{2}+2 \bar{\lambda}_{7} \bar{v}_{1}^{2} \bar{v}_{2}^{2}+\bar{\lambda}_{2} \bar{v}_{1} \bar{v}_{2}^{3}\right),
\end{aligned}
$$

а факторы в потенциале ${ }^{8)}$ определяются выражениями

$$
a_{1}=\frac{\sqrt{-\bar{\lambda}_{1}^{5}}}{52 \sqrt{6}}, \quad a_{2}=-\frac{\bar{\lambda}_{1}^{2}}{52}, \quad a_{3}=\frac{\sqrt{-\bar{\lambda}_{1}^{3}}}{13 \sqrt{6}}, \quad a_{4}=\frac{3 \bar{\lambda}_{1}}{52} .
$$

\section{5. АНАЛИЗ ЧИСЛЕННЫХ РЕЗУЛЬТАТОВ}

Для дальнейших численных оценок в рамках МССМ, относящихся к экспериментальным ограничениям на пространство параметров МССМ в свете данных коллайдера LHC, были использованы однопетлевые температурные поправки к константам взаимодействия четырех скалярных полей (13), полученные в работах [15]. Использовалась стандартная форма членов мягкого нарушения суперсимметрии [22]. Например, выражения для $\lambda_{1}$ и $\lambda_{6}$ имеют вид

$$
\begin{aligned}
\lambda_{1}=\frac{\bar{g}^{2}}{8} & +3 h_{t}^{4}|\mu|^{4} I_{2}\left[m_{Q}, m_{U}\right]+3 h_{b}^{4}\left|A_{b}\right|^{4} I_{2}\left[m_{Q}, m_{D}\right]+ \\
& +h_{t}^{2}|\mu|^{2}\left(-\frac{g_{1}^{2}-3 g_{2}^{2}}{2} I_{1}\left[m_{Q}, m_{U}\right]+2 g_{1}^{2} I_{1}\left[m_{U}, m_{Q}\right]\right)+ \\
& \quad+h_{b}^{2}\left|A_{b}\right|^{2}\left(\frac{12 h_{b}^{2}-g_{1}^{2}-3 g_{2}^{2}}{2} I_{1}\left[m_{Q}, m_{D}\right]+\left(6 h_{b}^{2}-g_{1}^{2}\right) I_{1}\left[m_{D}, m_{Q}\right]\right), \\
\lambda_{6}=- & 3 h_{t}^{4} \mu A_{t}|\mu|^{2} I_{2}\left[m_{Q}, m_{U}\right]-3 h_{b}^{4} \mu A_{b}\left|A_{b}\right|^{2} I_{2}\left[m_{Q}, m_{D}\right]+ \\
& +h_{t}^{2} \mu A_{t}\left(\frac{g_{1}^{2}-3 g_{2}^{2}}{4} I_{1}\left[m_{Q}, m_{U}\right]-g_{1}^{2} I_{1}\left[m_{U}, m_{Q}\right]\right)+ \\
& +h_{b}^{2} \mu A_{b}\left(\frac{-12 h_{b}^{2}+g_{1}^{2}+3 g_{2}^{2}}{4} I_{1}\left[m_{Q}, m_{D}\right]-\frac{6 h_{b}^{2}-g_{1}^{2}}{2} I_{1}\left[m_{D}, m_{Q}\right]\right),
\end{aligned}
$$

\footnotetext{
8) Называемые также математическими управляющими параметрами, в отличие от физических управляющих параметров $\lambda_{i}(T)$.
} 
где

$$
\begin{aligned}
& I_{1}\left[M_{a}, M_{b}\right]=-\frac{1}{64 \pi^{4} T^{2}} \int_{0}^{1} d x x \zeta\left[2, \frac{3}{2},\left(M_{a}^{2}-M_{b}^{2}\right) x+M_{b}^{2}\right], \\
& I_{2}\left[M_{a}, M_{b}\right]=\frac{3}{256 \pi^{6} T^{4}} \int_{0}^{1} d x x(1-x) \zeta\left[2, \frac{5}{2},\left(M_{a}^{2}-M_{b}^{2}\right) x+M_{b}^{2}\right]
\end{aligned}
$$

- интегралы обобщенной функции Гурвица $\zeta(u, s, t), g_{1}, g_{2}$ - константы связи калибровочных групп $U(1)$ и $S U(2), h_{t}, h_{b}$ - константы Юкавы CM, $\mu$ - параметр суперполя Хиггса, $A_{t}, A_{b}$ - трилинейные параметры взаимодействия суперпартнеров кварков с полями Хиггса в членах мягкого нарушения суперсимметрии, $m_{Q}$, $m_{U}, m_{D}$ - массовые параметры скалярных суперпартнеров кварков. Как правило, температурная эволюция взаимодействия четырех скалярных полей достаточно монотонная с характерной величиной изменения $\left|\lambda_{i}\right|$ от 0.1 до 1 на масштабе температур $10^{2}-10^{3}$ ГэВ [15].

В свете ненаблюдаемости дополнительных бозонов Хиггса в экспериментах LHC наиболее интересным представляется режим отщепления тяжелых бозонов, согласно которому остается только один относительно легкий скаляр на масштабе масс $M_{L}$, а остальные бозоны Хиггса имеют значительно большие массы на масштабе $M_{S} \gg M_{L}$. Режим отщепления реализуется, если параметры $\lambda_{i}$ по модулю не превосходят единицы, $m_{A} \gg\left|\lambda_{i}\right| v^{2}$ и $\cos ^{2}(\beta-\alpha) \approx \mathcal{O}\left(\left(v / M_{S}\right)^{4}\right), m_{h} \approx M_{L}=\mathcal{O}(v)$, $m_{H}, m_{A}, m_{H^{ \pm}} \approx M_{S}+\mathcal{O}\left(v^{2} / M_{S}\right)$ (подробнее см. [23]), причем все константы взаимодействия низкоэнергетической эффективной теории и массы частиц порядка $M_{L}$ остаются фиксированными. Условия $m_{H, A, H^{ \pm}} \approx M_{S} \geqslant 1$ ТэВ и $m_{h}=125$ ГэВ налагают ограничения на значения параметров МСCM. В дальнейшем будем использовать удовлетворяющий этим условиям набор $\left(\mu, m_{H^{ \pm}}, A\right)=(1.4,1,1.1 \mathrm{~T} э \mathrm{~B})^{9)}$. Режим отщепления для него показан на рис. 2. Интересно отметить, что при увеличении температуры массы тяжелых частиц меняются весьма слабо, а масса легкого бозона Хиггса $m_{h}$ существенно возрастает.

Отметим, что трилинейные параметры $A_{t}, A_{b}$ членов мягкого нарушения суперсимметрии, параметр хиггсовского суперполя $\mu$, а также массовые параметры сектора суперпартнеров кварков $m_{Q}, m_{U}$ и $m_{D}$, использованные для построения зависимостей на рис. 2, выбраны в духе так называемого "естественного" сценария MCCM [24], который в настоящее время является базовым для построения контуров исключения в пространстве параметров МССМ при моделировании каналов распада суперпартнеров коллаборациями CMS и ATLAS. B рамках "естественного" сценария хиггсино (например, чарджино и два нейтралино) и суперпартнеры кварков $\tilde{t}_{R}$, $\tilde{t}_{L}, \tilde{b}_{L}$ имеют массы порядка $10^{2}$ ГэВ и небольшие расщепления масс (все скварки тяжелее хиггсино), глюино находится на масштабе $10^{3}$ ГэВ, а все остальные суперчастицы находятся на масштабе масс 10-100 ТэВ. Описанные выше различия между массами частиц МССМ предпочтительны для всех сценариев, где неестественная “точная настройка" параметров отсутствует, что позволяет облегчить проблему "малой иерархии масс". Масса легкого бозона МССМ $m_{h}=125.02$ ГэВ соответствует

9) Данный набор значений, вообще говоря, представляет собой граничные условия для зависящих от температуры функций $\mu(T), m_{H^{ \pm}}(T), A(T)$, однако в силу их слабой температурной зависимости в данной работе набор фиксирован. 


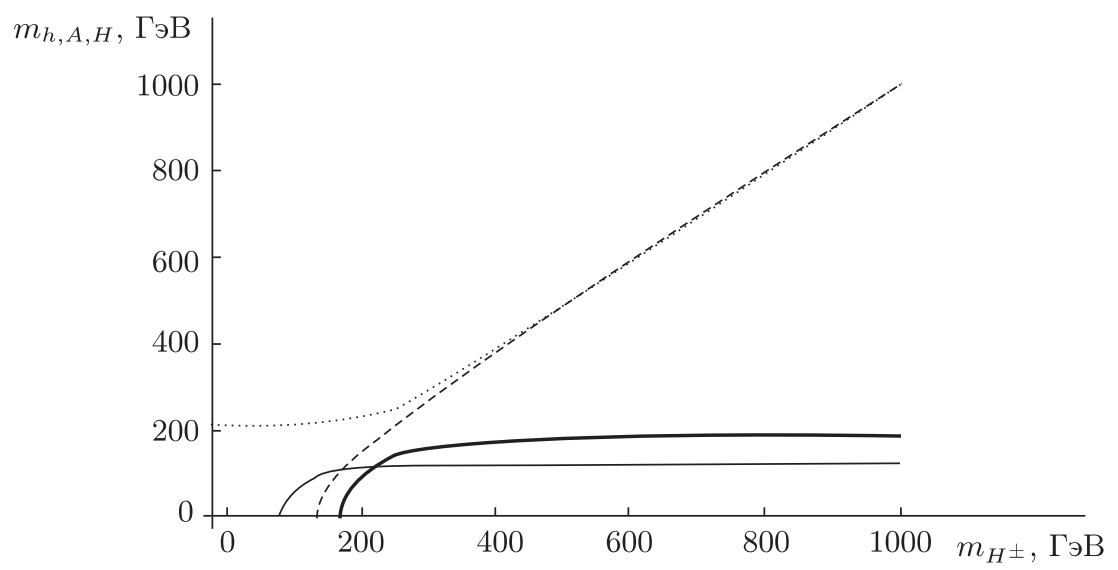

Рис. 2. Зависимость масс бозонов Хиггса от $m_{H^{ \pm}}$при $T=0$ и при $T=$ 120 ГэВ: $m_{h}(0)$ - тонкая сплошная линия; $m_{h}(120)$ - сплошная линия; $m_{A}$ (штриховая линия) и $m_{H}$ (пунктирная линия) слабо зависят от $T$.

большим значениям трилинейных параметров (более 1 ТэВ) и умеренным вырожденным $m_{Q}=m_{U}$ с допустимыми минимальными значениями 100-300 ГэВ (в зависимости от деталей структуры расщепления масс). Роль наиболее легкой суперсимметричной частицы (LSP) играет хиггсино. Экспериментальные ограничения на массы тяжелых бозонов Хиггса MCСМ зависят от используемого параметрического сценария.

Например, последние ограничения коллаборации CMS в каналах распада $\tau^{+} \tau^{-}$ представлены [25] в виде контуров исключения на плоскости $\left(m_{A}, \operatorname{tg} \beta\right)$ для семи параметрических сценариев. В рамках интересующего нас "естественного" сценария MCCM, по всей видимости, существуют две возможности: либо большие значения $\operatorname{tg} \beta \sim 50$ в комбинации с $m_{A} \sim 1$ ТэВ, либо малые величины $\operatorname{tg} \beta \sim 1$ в комбинации с $m_{A} \sim 300-500$ ГэВ. Параметр хиггсовского суперполя $\mu$ должен быть всегда положительным и достаточно большим (порядка 1 ТэВ), поскольку радиационная поправка, "дотягивающая" массу легкого бозона Хиггса от $m_{Z}$ ("древесная" масса в MCCM на масштабе масс суперпартнеров $M_{\mathrm{MSSM}}$ ) до 125.02 ГэВ, определяется отношениями $\mu / M_{\mathrm{MSSM}}, A_{t, b} / M_{\mathrm{MSSM}}$ (см. явные выражения для $\lambda_{i}$ в [12]).

Таким образом, рассматриваемый нами "предел отщепления" согласуется, по крайней мере качественно, с ограничениями, полученными на основе анализа экспериментальных данных в рамках "естественного" сценария МССМ. Допустимые пределы изменения $\operatorname{tg} \beta(0)$ составляют от нескольких единиц до 50. Верхний предел $\operatorname{tg} \beta(0) \sim 50$ возникает при учете экспериментального ограничения на сечение парного рождения $t \bar{t}$ с последующим распадом топ-кварка $t \rightarrow H^{ \pm} b$, которое усилено фактором $\operatorname{tg}^{4} \beta(0)$, становящимся неприемлемо большим уже при энергиях коллайдера Tevatron. Интерес к области значений $\operatorname{tg} \beta(0) \sim 1$ связан со сценариями легкого суперпартнера топ-кварка и слабой связи бозонов Хиггса с $\tau$-лептоном [26]. Отметим, что описанная выше схема для масштабов масс суперпартнеров в рамках "естественного" сценария при малом $\operatorname{tg} \beta(0)$ предпочтительна также для феноменологии HMCCM [27]. 


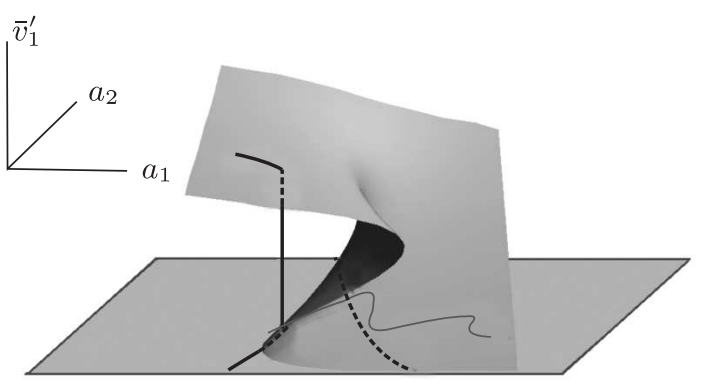

Рис. 3. Многообразие катастрофы типы $A_{3}$ : кривая на поверхности многообразия - траектория системы, штриховая кривая - сепаратриса.

В рамках рассматриваемого нами наиболее общего случая эволюции, когда угол $\beta$ меняется произвольно, $\beta(0) \neq \beta\left(T_{\mathrm{c}}\right)$. Для численных оценок значение $\beta\left(T_{\mathrm{c}}\right)$ примем равным 5 или 50. Согласно критерию Шапошникова [28] $v_{\mathrm{c}}=T_{\mathrm{c}}$. На рис. 3 приведена качественная картина многообразия катастрофы типа $A_{3}$ (так называемая катастрофа сборки) в пространстве $\left(a_{1}, a_{2}, \bar{v}_{1}^{\prime}\right)$. Эволюции состояния системы в зависимости от температуры соответствует линия на поверхности многообразия. Бифуркационное множество - сепаратриса - функции-катастрофы

$$
\left(-\frac{a_{2}}{6}\right)^{3}=\left(\frac{a_{1}}{8}\right)^{2}
$$

изображено штриховой кривой в плоскости $\left(a_{1}, a_{2}\right)$. Значение $\bar{v}_{1}^{\prime}$, равное нулю до пересечения бифуркационного множества, в момент его пересечения скачкообразно меняется на $\bar{v}_{1}^{\prime}\left(T_{\mathrm{c}}\right)$ при критической температуре $T_{\mathrm{c}}$.

Численная оценка $a_{1}, a_{2}$ модельно-зависима по отношению к значениям $\beta_{\mathrm{c}}, m_{Q}$, $m_{U}, m_{D}$. Например, уменьшение параметра $\beta_{\mathrm{c}}$ приводит к понижению $T_{\mathrm{c}}$. На рис. 4 изображены температурные зависимости сепаратрисы (37) и управляющих параметров (31) при $\operatorname{tg} \beta=5$. Видно, что система проходит через бифуркационное множество, и, следовательно, происходит фазовый переход при $T_{\mathrm{c}}=58$ ГэВ. В то время как при изменении температуры форма потенциала (30) вдоль оси $\bar{v}_{2}^{\prime}$ характеризуется устойчивым минимумом, вдоль оси $\bar{v}_{1}^{\prime}$ соответствующая форма неморсовской части потенциала существенно меняется. Так, при температуре выше $T_{\text {с }}$ система имеет два физических минимума, один из которых по мере остывания вещества становит-

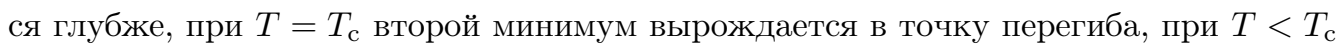
система имеет один минимум (рис.5). На рис. 6 в плоскости управляющих параметров $\left(a_{1}, a_{2}\right)$ представлена сепаратриса катастрофы типа $A_{3}(37)$ и траектория движения системы при углах $\operatorname{tg} \beta_{\mathrm{c}}=5$ и $\operatorname{tg} \beta_{\mathrm{c}}=50$ соответственно. Видно, что при $T \sim 200$ ГэВ система находится в области сборки многообразия катастрофы (нижний лист на рис. 3), потенциал имеет два минимума и, вообще говоря, возможен переход вакуумного конденсата в любой точке этой области. Будем, однако, придерживаться "принципа максимального промедления Максвелла", согласно которому скачок происходит лишь при пересечении траекторией системы дальней ветви сепаратрисы. В нашем случае скачок происходит при $T_{\mathrm{c}}=58$ ГэВ или при $T_{\mathrm{c}}=62$ ГэВ при 


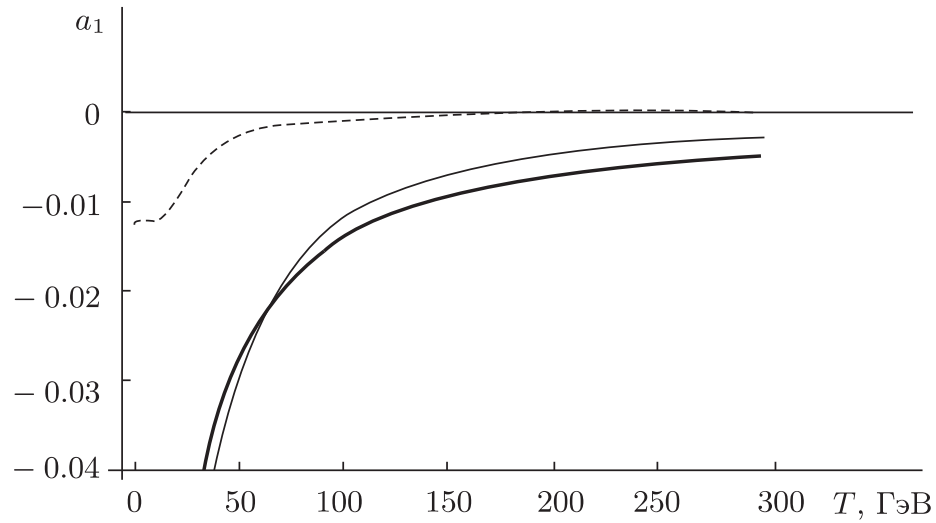

Рис. 4. Зависимость сепаратрисы (жирная сплошная кривая) и управляющих параметров $a_{i}$ от температуры ( $a_{1}$ - тонкая сплошная кривая, $a_{2}-$ штриховая кривая).

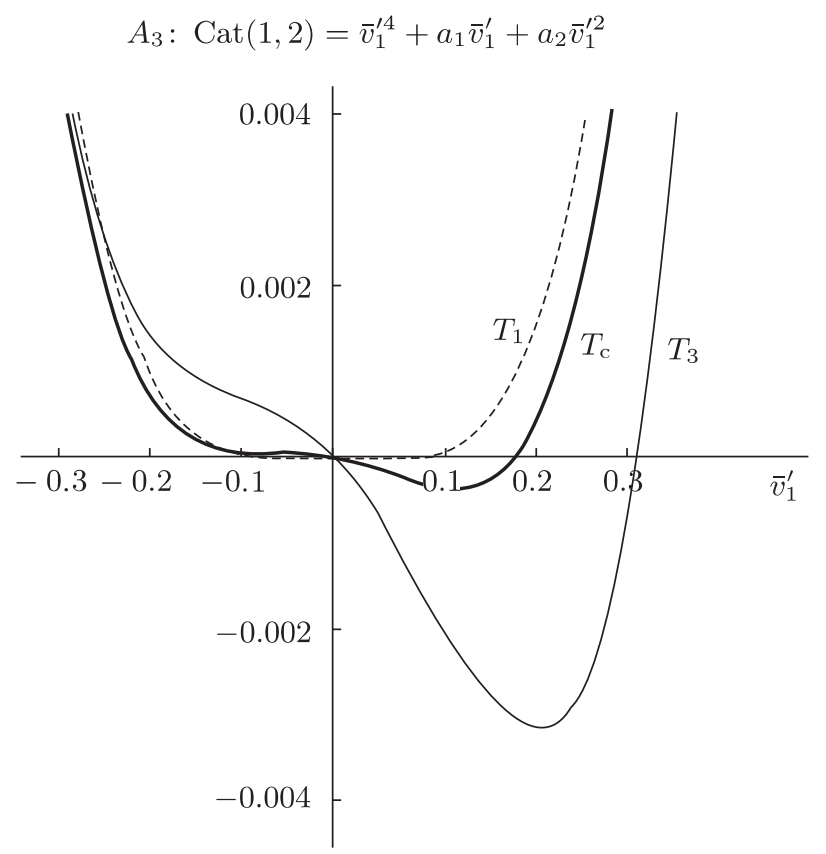

Рис. 5. Вид неморсовской части потенциала (30) при $T_{1}=220$ ГэВ, $T_{\mathrm{c}}=$ $58 \Gamma \ni \mathrm{B}, T_{3}=10$ ГэВ.

$\operatorname{tg} \beta_{\mathrm{c}}=5$ и $\operatorname{tg} \beta_{\mathrm{c}}=50$ соответственно. Из рис. 6 также видно, что с понижением температуры параметры $a_{1}, a_{2}$ могут как возрастать (рис. 6а), так и убывать (рис. 6б). На траектории системы для наглядности точками отмечены состояния, для которых изображена функция $\operatorname{Cat}(1,2)$. 


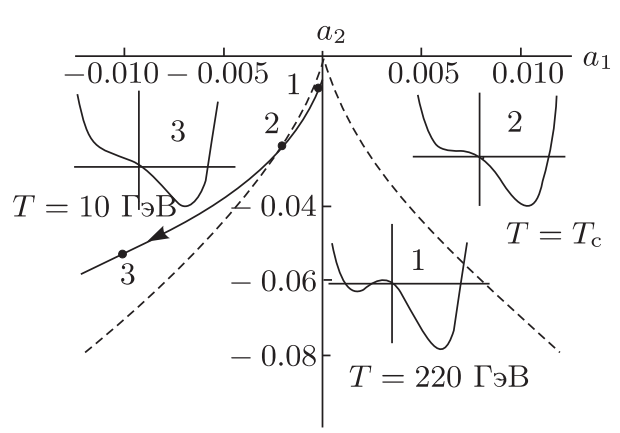

a

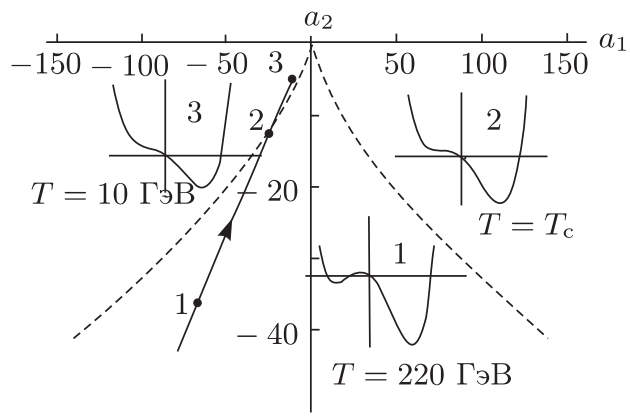

б

Рис. 6. Сепаратриса (штриховая линия) и траектория системы (сплошная линия) в плоскости $\left(a_{1}, a_{2}\right)$ при $\operatorname{tg} \beta_{\mathrm{c}}=5$ (а) и $\operatorname{tg} \beta_{\mathrm{c}}=50$ (б). Не указанный явно масштаб по вертикальной оси для шести кривых, иллюстрирующих форму потенциала $\bar{U}$, на два порядка меньше масштаба, который обозначен на рис. 5. Для численной оценки взяты значения $g_{1}=0.3573, g_{2}=0.6517, h_{t}=1$, $h_{b}=0.1, \mu=1.4 \mathrm{T \ni B}, v\left(T_{\mathrm{c}}\right)=T_{\mathrm{c}}, m_{t}=175$ ГэВ, $m_{b}=3$ ГэВ, $m_{Q}=100$ ГэВ, $m_{U}=100$ ГэВ, $m_{D}=800$ ГэВ.

Многообразие катастрофы типа $A_{5}$ (так называемая катастрофа бабочки) для общего случая больших радиационных поправок в МССМ задается в пятимерном пространстве $\left(a_{1}, a_{2}, a_{3}, a_{4}, \bar{v}_{1}^{\prime}\right)$. В общем случае пространство $\left(a_{1}, a_{2}, a_{3}, a_{4}\right)$ разбивается на открытые области, внутри которых поведение функции (32) качественно одинаково, оно изменяется только при прохождении системы через поверхность сепаратрисы. Численные оценки для этого случая более сложные и выходят за рамки настоящей работы.

\section{6. ЗАКЛЮЧЕНИЕ}

В настоящей работе рассматривается перемещение экстремума двухдублетного потенциала Хиггса по поверхности равновесия вдоль произвольной траектории по мере уменьшения температуры от некоторого ее значения в симметричной фазе теории до нуля при наблюдаемом в настоящее время значении вакуумного среднего $v(0)=246$ ГэВ. В этом заключается основное отличие нашего исследования от анализов, использующих одномерную эволюцию потенциальной функции, в рамках которых, в частности, фазовый переход первого рода появляется вследствие неаналитичности интеграла в выражении для плотности свободной энергии по переменной $m^{2} / T^{2}$. В рассматриваемом подходе температурная зависимость содержится в управляющих параметрах $\lambda_{i}(T)$ и вычисляется для каждой диаграммы в рамках формализма мнимого времени [15].

В настоящей работе предполагается, что эффективный потенциал (1) является первичным объектом, подлежащим калибровочной $S U(2)$-симметрии, а не морсифицированной функцией-катастрофой. В рамках теории катастроф удобно исследовать эволюцию минимума эффективного потенциала (1) при произвольном изменении управляющих параметров $\lambda_{i}$, зависящих от температуры и энергии, когда 
наглядная картина для одномерной зависимости потенциала от температуры (стандартная модель) или же картина перемещения экстремума по поверхности равновесия отсутствует. Наличие гладких нелинейных преобразований, приводящих потенциал (4) к каноническим формам для изолированной и неизолированной критических точек, существенно для стабильности полученных результатов при включении достаточно малых осцилляций полей $\eta_{i}, \chi_{i}$ в калибровочном базисе вблизи вакуумных средних $v_{1}, v_{2}$. "Шевеление поверхности равновесия" (или многообразия катастрофы) при таком включении не меняет существенно результаты.

В окрестности критических точек потенциал Хиггса, зависящий от не более чем трех переменных состояния и от менее чем пяти управляющих параметров, с заданной степенью точности приводится нелинейными преобразованиями для вакуумных конденсатов к каноническим формам, свойства которых в общем случае хорошо изучены. Исходный потенциал $U\left(v_{1}, v_{2}\right)$ обладает $S U(2) \times U(1)$-симметрией; нелинейное осесохраняющее преобразование $\left(v_{1}, v_{2}\right) \rightarrow\left(\bar{v}_{1}^{\prime}, \bar{v}_{2}^{\prime}\right)$ справедливо вблизи окрестности критической точки, и новая система координат является локальной. Скачкообразное изменение вакуумных средних, характерное для фазовых переходов первого рода, для распространенных моделей двухдублетного хиггсовского сектора описывается функциями-катастрофами типа $A_{3}$ (два управляющих параметра) или типа $A_{5}$ (четыре управляющих параметра).

Качественный анализ катастрофы типа $A_{3}$ (катастрофа сборки) при реалистических значениях переменных пространства параметров МССМ показывает, что при температурах вблизи 60 ГэВ вакуумное ожидание $\bar{v}_{2}^{\prime}$, как правило, меняется плавно, в то время как $\bar{v}_{1}^{\prime}$ переходит скачком из симметричной фазы $(0 ; 0)$ в фазу нарушенной симметрии $\left(\bar{v}_{1}^{\prime c} ; \bar{v}_{2}^{\prime c}\right)$, после чего оба вакуумных конденсата плавно меняются при понижении температуры и достигают современного значения $v=246$ ГэВ. "Естественный" параметрический сценарий MCCM [24] с отщеплением состояний гейджино адекватен фазовому переходу первого рода на "горячей стадии" эволюции Вселенной.

Благодарности. Работа частично поддержана Программой поддержки ведущих научных школ (грант НШ-3042.2014.2).

\section{Список литературы}

[1] S. Weinberg, Phys. Rev. D, 9:12 (1974), 3357-3378; A. D. Linde, Rep. Prog. Phys., 42:3 (1979), 389-437.

[2] В. А. Рубаков, М.Е. Шапошников, УФН, 166:5 (1996), 493-537; Д. С. Горбунов, В. А. Рубаков, Введение в теорию ранней Вселенной. Теория горячего Большого взрива, ЛКИ, М., 2008.

[3] G. Aad, T. Abajyan, B. Abbott et al. [ATLAS Collaboration], Phys. Lett. B, 716:1 (2012), 1-29, arXiv: 1207.7214; S. Chatrchyan, V. Khachatryan, A. M. Sirunyan et al. [CMS Collaboration], Phys. Lett. B, 716:1 (2012), 30-61, arXiv: 1207.7235.

[4] E. Boos, V. Bunichev, M. Dubinin, Y. Kurihara, Phys. Rev. D, 89:3 (2014), 035001, 12 pp., arXiv: 1309.5410.

[5] D. Lyth, A. Riotto, Phys. Rep., 314:1-2 (1999), 1-146, arXiv: hep-ph/9807278.

[6] A. D. Linde, Phys. Lett. B, 108:6 (1982), 389-393.

[7] A. D. Linde, Phys. Lett. B, 129:3-4 (1983), 177-181; A. Albrecht, P. J. Steinhardt, Phys. Rev. Lett., 48:17 (1982), 1220-1223. 
[8] A. Linde, Phys. Rev. D, 49:2 (1994), 748-754, arXiv: astro-ph/9307002.

[9] F. Bezrukov, J. Rubio, M. Shaposhnikov, Living beyond the edge: Higgs inflation and vacuum metastability, arXiv: 1412.3811; F. Bezrukov, M. Shaposhnikov, Phys. Lett. B, 734 (2014), 249-254, arXiv: 1403.6078; F. Bezrukov, Class. Quantum Grav., 30:21 (2013), 214001, 21 pp., arXiv: 1307.0708.

[10] L. E. Ibanez, I. Valenzuela, Phys. Lett. B, 736 (2014), 226-230, arXiv: 1404.5235; S. Kasuya, T. Moroi, F. Takahashi, Phys. Lett. B, 593:1-4 (2004), 33-41, arXiv: hep-ph/0312094.

[11] M. Carena, G. Nardini, M. Quirós, C.E. M. Wagner, Nucl. Phys. B, 812:1-2 (2009), 243-263, arXiv: 0809.3760; L. Fromme, S. J. Huber, M. Seniuch, JHEP, 11 (2006), 038, 25 pp., arXiv: hep-ph/0605242; C. Balazs, M. Carena, A. Menon, D. Morrissey, C.E. M. Wagner, Phys. Rev. D, 71:7 (2005), 075002, 16 pp., arXiv: hep-ph/0412264; S. Kanemura, Y. Okada, E. Senaha, "Electroweak baryogenesis and quantum corrections to the Higgs potential", Workshop on CP Studies and Non-Standard Higgs Physics, CERN-2006-009 (May 2004 - December 2005), eds. S. Kraml, G. Azuelos, D. Dominici, J. Ellis, G. Grenier, H.,E. Haber, J. S. Lee, D. J. Miller, A. Pilaftsis, W. Porod, CERN, Geneva, 2006, 41-44, arXiv: hep-ph/0608079; J. Cline, K. Kainulainen, Nucl. Phys. B, 510:1-2 (1998), 88-102, arXiv: hep-ph/9705201; G. R. Farrar, M. Losada, Phys. Lett. B, 406:1-2 (1997), 60-65, arXiv: hep-ph/9612346; P. Arnold, O. Espinosa, Phys. Rev. D, 47:8 (1993), 3546-3579; 50:10 (1994), 6662, arXiv: hep-ph/9212235 Erratum; A. Brignole, J. R. Espinosa, M. Quirós, F. Zwirner, Phys. Lett. B, 324:2 (1994), 181-191, arXiv: hep-ph/9312296; G.F. Giudice, Phys. Rev. D, 45:9 (1992), 3177-3182; N. Turok, J. Zadrozny, Nucl. Phys. B, 369:3 (1992), 729-742; A.I. Bochkarev, S. V. Kuzmin, M. E. Shaposhnikov, Phys. Lett. B, 244:2 (1990), 275-278.

[12] E. Akhmetzyanova, M. Dolgopolov, M. Dubinin, Phys. Rev. D, 71:7 (2005), 075008, 24 pp., arXiv: hep-ph/0405264; Э. Н. Ахметзянова, М. В. Долгополов, М. Н. Дубинин, ЯФ, 70:9 (2007), 1594-1597.

[13] M. N. Dubinin, A. V. Semenov, Eur. J. Phys. C, 28:2 (2003), 223-236, arXiv: hep-ph/0206205; F. Boudjema, A. Semenov, Phys. Rev. D, 66:9 (2002), 095007, 15 pp., arXiv: hep-ph/0201219.

[14] E. Akhmetzyanova, M. Dolgopolov, M. Dubinin, "Self-couplings of Higgs bosons in scenarios with mixing of CP-even/CP-odd states", Workshop on CP Studies and Non-Standard Higgs Physics, CERN-2006-009 (May 2004 - December 2005), eds. S. Kraml, G. Azuelos, D. Dominici, J. Ellis, G. Grenier, H.,E. Haber, J. S. Lee, D. J. Miller, A. Pilaftsis, W. Porod, CERN, Geneva, 2006, 133-138, arXiv: hep-ph/0608079.

[15] M. Dolgopolov, M. Dubinin, E. Rykova, J. Modern Phys., 2:5 (2011), 301-322, arXiv: 0901.0524; А. О. Борисов, М. В. Долгополов, М. Н. Дубинин, Э. Н. Рыкова, ЯФ, 72:1 (2009), 175-180.

[16] R. Gilmore, Catastrophe Theory for Scientists and Engineers, Dover, New York, 1993; В. И. Арнольд, УМH, 30:5(185) (1975), 3-65; R. Thom, Structural Stability and Morphogenesis, Reading, MA, Benjamin, 1975; M. Morse, Trans. Amer. Math. Soc., 33:1 (1931), $72-91$.

[17] K. Funakubo, S. Tao, F. Toyoda, Prog. Theor. Phys., 109:3 (2003), 415-432, arXiv: hep-ph/0211238; M. Brhlik, G. J. Good, G. L. Kane, Phys. Rev. D, 63:3 (2001), 035002, 8 pp., arXiv: hep-ph/9911243; M. Laine, K. Rummukainen, Higgs sector CP-violation at the electoroweak phase transition, arXiv: hep-ph/9811369.

[18] R. D. Peccei, H. R. Quinn, Phys. Rev. Lett., 38:25 (1977), 1440-1443.

[19] S. Weinberg, Phys. Rev. Lett., 40:4 (1978), 223-226.

[20] S. Davidson, H. Haber, Phys. Rev. D, 72:3 (2005), 035004, 21 pp., arXiv: hep-ph/0504050.

[21] М. Н. Дубинин, Е. Ю. Петрова, Канонические формы теории катастроф для потенциала Хиггса двухдублетной модели, Препринт № 2014-3/887, НИИЯФ МГУ, М., 2014, Электронный ресурс: http://www.sinp.msu.ru/ru/preprints/. 
[22] H. Haber, R. Hempfling, Phys. Rev. D, 48 (1993), 4280-4309, arXiv: hep-ph/9307201.

[23] Э. Н. Ахметзянова, М. В. Долгополов, М. В. Дубинин, ЭЧАЯ, 37:5 (2006), 1285-1382.

[24] M. Papucci, J. Ruderman, A. Weiler, JHEP, 09 (2012), 035, 45 pp., arXiv: 1110.6926;

T. Cherghetta, B. von Harling, N. Setzer, JHEP, 07 (2011), 011, 35 pp., arXiv: 1104.3171;

N. Craig, D. Green, A. Katz, JHEP, 07 (2011), 045, 32 pp., arXiv: 1103.3708.

[25] V. Khachatryan, A. M. Sirunyan, A. Tumasyan et al. [CMS Collaboration], JHEP, 10 (2014), 160, 53 pp., arXiv: 1408.3316; S. Chatrchyan, V. Khachatryan, A. M. Sirunyan et al. [CMS Collaboration], Phys. Rev. Lett., 106:23 (2011), 231801, 15 pp., arXiv: 1104.1619.

[26] M. Carena, S. Heinemeyer, O. Stal, G. Weiglein, Eur. Phys. J. C, 73 (2013), 2552-2571.

[27] S. F. King, M. Muhlleitner, R. Nevzorov, K. Walz, Nucl. Phys. B, 870:2 (2013), 323-352.

[28] М.Е. Шапошников, Писъма в ЖКЭТФ, 44:8 (1986), 364-366.

Поступила в редакцию 8.11.2014, после доработки 16.02.2015 